KANAZAWA-05-15

\title{
Nonabelian Discrete Family Symmetry to Soften the SUSY Flavor Problem and to Suppress Proton Decay
}

\author{
Yuji Kajiyama, Etsuko Itou, and Jisuke Kubo \\ Institute for Theoretical Physics, Kanazawa University, Kanazawa 920-1192, Japan
}

\begin{abstract}
Family symmetry could explain large mixing of the atmospheric neutrinos. The same symmetry could explain why the flavor changing current processes in supersymmetric standard models can be so suppressed. It also may be able to explain why the proton is so stable. We investigate these questions in a supersymmetric, renormalizable extension of the standard model, which possess a family symmetry based on a binary dihedral group $Q_{6}$. We find that the amplitude for $\mu \rightarrow e+\gamma$ enjoys a suppression factor proportional to $\left|\left(V_{\mathrm{MNS}}\right)_{e 3}\right| \simeq m_{e} /\left(\sqrt{2} m_{\mu}\right) \simeq 3.4 \times 10^{-3}$, and that $B(p \rightarrow$ $\left.K^{0} \mu^{+}\right) / B\left(p \rightarrow K^{0} e^{+}\right) \simeq\left|\left(V_{\mathrm{MNS}}\right)_{e 3}\right|^{2} \simeq 10^{-5}$, where $V_{\mathrm{MNS}}$ is the neutrino mixing matrix.
\end{abstract}

PACS numbers: 12.60.Jv,11.30.Hv, 12.15.Ff, 14.60.Pq, 02.20.Df 


\section{INTRODUCTION}

The remarkable success of the standard model (SM) suggests that we have a highly nontrivial part of a more fundamental theory for elementary particle physics. In spite of this success, the SM suffers from various problems. One of them is that due to the quadratic divergence of the Higgs mass its natural scale can not exceed $O(1) \mathrm{TeV}$ 1]. Therefore, in order to extend the SM in a natural way, the quadratic divergence has to be cancelled 1, 2]. As it is well known today, low energy supersymmetry (SUSY) is introduced to protect the Higgs mass from the quadratic divergence [3, 4]. Unfortunately, SUSY is broken, and therefore its breaking should be soft to maintain the very nature of low energy SUSY, whatever its origin is [3, 4].

Renormalizability allows an introduction of a certain set of soft supersymmetry breaking (SSB) parameters. In the minimal supersymmetric standard model (MSSM), more than 100 SSB parameters can be introduced [5]. Moreover, these parameters should be highly fine tuned so that they do not induce unacceptably large flavor changing neutral currents (FCNCs) and CP violations 6, 7, 8, 9, 10]. Why should they be so fine tuned? What controls them ? This is the so-called SUSY flavor problem, which has existed ever since SUSY found phenomenological applications 11].

There are several theoretical approaches to overcome this problem [3, 4] [12]- 25]. One of them is to base on a family symmetry [22]-[25]. However, if the family symmetry is hardly broken (i.e. broken by operators with dimension $\geq 4$ ), non-symmetric SSB terms can be generated in higher orders in perturbation theory. Then it is not possible to make quantitative statements on the induced non-symmetric SSB terms, because these terms are infinite in perturbation theory, however small the hard breaking terms are. In this paper, we consider a supersymmetric model, recently proposed in 26], which posses a nonabelian discrete family symmetry based on a binary dihedral group $Q_{6}$. We investigate how the SUSY flavor problem can be softened by $Q_{6}$ family symmetry, which is our first task. We find that except for few cases the fine tuning of the SSB parameters is not necessary in this model. The only observable process would be $\mu \rightarrow e+\gamma$, whose branching fraction enjoys a suppression factor proportional to $\left|\left(V_{\mathrm{MNS}}\right)_{e 3}\right|^{2} \simeq m_{e}^{2} /\left(2 m_{\mu}^{2}\right) \simeq 10^{-5}$, and is three (four) orders of magnitude larger than that of $\tau \rightarrow e(\mu)+\gamma$ in the model, where $V_{\mathrm{MNS}}$ is the neutrino mixing matrix.

In the MSSM without $R$ parity invariance, there exist about 100 dimension-four operators that violate the baryon number or lepton number conservations. Fortunately, $R$ invariance can kill all these dangerous dimension-four operators, while it together with $B-L$ conservation allows two types of dimension-five operators that lead to proton decay [27, 28]. It is therefore widely expected that these operators are responsible for an observable proton decay. Indeed, in the minimal SUSY grand unified theory (GUT) based on $S U(5)$ [1]], these operators force protons to decay faster than the experimental bound [29]. Given a family symmetry, the flavor structure is fixed, at least partially, and it can control the flavor 
changing effects in proton decay, too [30]. In this paper we assume that some unknown Planck scale physics, which respects $Q_{6}$ family symmetry, induces dimension-five operators that lead to proton decay. We first would like to find out all $B$ and $L$ violating operators with dimension $\leq 5$ allowed by $Q_{6}$, and then to investigate how the family symmetry acts on proton decay. If we assume the degeneracy of the scalar quark masses, then the branching fraction $B\left(p \rightarrow K^{0} e^{+}\right)$is similar to that of [30] that it becomes comparable with $B\left(p \rightarrow K^{+} \bar{\nu}\right)$. If the degeneracy assumption is dropped, $B\left(p \rightarrow K^{0} e^{+}\right)$can become $O\left(10^{-4}\right) \times B\left(p \rightarrow K^{+} \bar{\nu}\right)$, which is still two orders of magnitude lager than that of the minimal SUSY GUT case. It turns out that in contrast to the case of [30] the branching fraction $B\left(p \rightarrow K^{0} \mu^{+}\right)$, which turns out to be proportional to $\left|\left(V_{\mathrm{MNS}}\right)_{e 3}\right|^{2}$, is five orders of magnitude smaller than $B\left(p \rightarrow K^{0} e^{+}\right)$in the present case, irrespective of the degeneracy of the scalar quark masses. The main reason for this is the maximal mixing in the charged lepton sector, which serves the maximal mixing of the atmospheric neutrinos in this model. We also give an upper bound on the coupling constants that are assumed to be generated by Planck scale physics. We find that the family symmetry can raise the upper bound by two orders of magnitude.

In sect. II we outline the model of [26]. Because of the nonabelian family symmetry, the Yukawa sector of the model is strongly constrained, and the redundancy of the parameters of this sector is significantly reduced. As a consequence, we can explicitly give the unitary matrices, $U_{L}$ and $U_{R}$, that rotate the left and right-handed fermions to diagonalize the corresponding mass matrices. They are needed to quantitatively discuss the SUSY flavor problem in the super CKM basis in sect. III. For completeness, we re-present the predictions from $Q_{6}$ family symmetry. In sect. IV we consider the $B$ and $L$ violating operators that are allowed by the family symmetry and then calculate the dominant proton decay modes as function of superpartner masses. We conclude in sect. V.

\section{THE MODEL}

\section{A. Group theory of $Q_{2 N}$}

The binary dihedral group $Q_{2 N}(N=2,3, \ldots)$ is a finite subgroup of $S U(2)^{1}$.

Its defining matrices are given by [26, 31]

$$
\tilde{R}_{2 N}=\left(\begin{array}{cc}
\cos \frac{\theta_{N}}{2} & \sin \frac{\theta_{N}}{2} \\
-\sin \frac{\theta_{N}}{2} & \cos \frac{\theta_{N}}{2}
\end{array}\right), \tilde{P}_{Q}=\left(\begin{array}{cc}
i & 0 \\
0 & -i
\end{array}\right),
$$

where $\theta_{N}=2 \pi / N$. Then the set of $4 N$ elements of $Q_{2 N}$ is given by

$$
\mathcal{G}_{Q_{2 N}}=\left\{\tilde{R}_{2 N},\left(\tilde{R}_{2 N}\right)^{2}, \ldots,\left(\tilde{R}_{2 N}\right)^{2 N}=\mathbf{1}, \tilde{R}_{2 N} \tilde{P}_{Q},\left(\tilde{R}_{2 N}\right)^{2} \tilde{P}_{Q}, \ldots,\left(\tilde{R}_{2 N}\right)^{2 N} \tilde{P}_{Q}=\tilde{P}_{Q}\right\}
$$

\footnotetext{
${ }^{1}$ Models based on dihedral flavor symmetries, ranging from $D 3\left(\simeq S_{3}\right)$ to $Q_{6}$ and $D_{7}$, have been recently discussed in [26], 31]- 37 ].
} 
Only one- and two-dimensional irreps. exist. There exist four different one-dimensional irreps of $Q_{2 N}$, which can be characterized according to $Z_{2} \times Z_{4}$ charge:

$$
\begin{aligned}
& \mathbf{1}_{+, 0}, \quad \mathbf{1}_{-, 0}, \mathbf{1}_{+, 2}, \mathbf{1}_{-, 2} \text { for } N=2,4,6, \ldots, \\
& \mathbf{1}_{+, 0}, \quad \mathbf{1}_{-, 1}, \mathbf{1}_{+, 2}, \mathbf{1}_{-, 3} \text { for } N=3,5,7, \ldots \text {, }
\end{aligned}
$$

where the $\mathbf{1}_{+, 0}$ is the true singlet of $Q_{2 N}$, and only $\mathbf{1}_{-, 1}$ and $\mathbf{1}_{-, 3}$ are complex irreps. The $N-1$ different two-dimensional irreps are denoted by

$$
\mathbf{2}_{\ell}, \quad \ell=1, \ldots, N-1
$$

$\mathbf{2}_{\ell}$ with odd $\ell$ is a pseudo real representation, while $\mathbf{2}_{\ell}$ with even $\ell$ is a real representation. It is straightforward to calculate the Clebsch-Gordan coefficients for tensor products of irreps. The following multiplication rules in $Q_{6}$ in particular are used to construct the model [26].

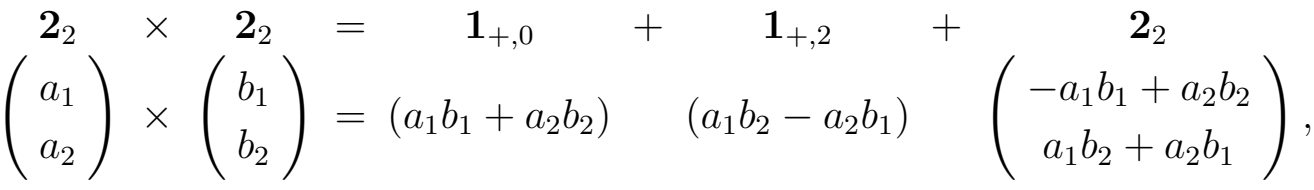

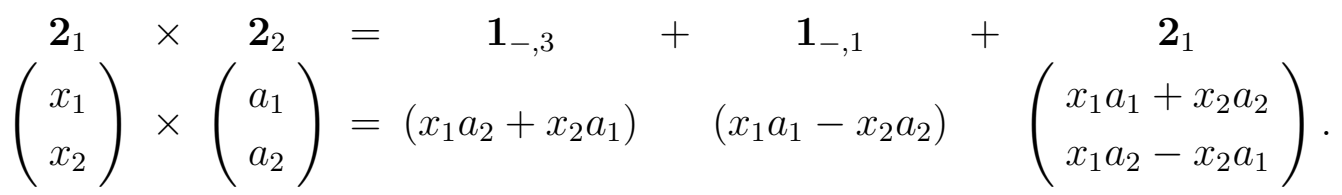

\section{B. $\quad Q_{6}$ assignment and superpotential}

In Table \ we write the $Q_{6}$ assignment of the quark, lepton and Higgs chiral supermultiplets $^{2}$, where $Q, Q_{3}, L, L_{3}$ and $H^{u}, H_{3}^{u}, H^{d}, H_{3}^{d}$ stand for $S U(2)_{L}$ doublets supermultiplets for quarks, leptons and Higgs bosons, respectively. Similarly, $S U(2)_{L}$ singlet supermultiplets for quarks, charged leptons and neutrinos are denoted by $U^{c}, U_{3}^{c}, D^{c}, D_{3}^{c}, E^{c}, E_{3}^{c}$ and $N^{c}, N_{3}^{c}$. $S, T$ and $Y$ are $S U(3)_{C} \times S U(2)_{L} \times U(1)_{Y}$ singlet Higgs supermultiplets. As an alterative

to the abelian discrete $R$ symmetry $Z_{12 R}$ of [26], for which one needs more singlets to construct a desired Higgs sector, we introduce an abelian $Z_{4}$ symmetry. The $Z_{4}$ constrains the Higgs sector, where it does not constrain anything in the Yukawa sector. When discussing proton decay in sect. IV, we will assume neither $Z_{4}$ nor $Z_{12 R}$, because the symmetry of the Higgs sector may have a stronger model dependence. We then write down the most general, renormalizable, $Q_{6} \times Z_{4} \times R$ invariant superpotential $W$ :

$$
W=W_{Q}+W_{L}+W_{H}
$$

\footnotetext{
${ }^{2}$ The same model exists for $Q_{2 N}$ if $N$ is odd and a multiple of 3 .
} 


\begin{tabular}{|c|c|c|c|c|c|c|c|c|c|c|c|c|c|c|}
\hline & $Q$ & $Q_{3}$ & $U^{c}, D^{c}$ & $U_{3}^{c}, D_{3}^{c}$ & $L$ & $L_{3}$ & $E^{c}, N^{c}$ & $E_{3}^{c}$ & $N_{3}^{c}$ & $H^{u}, H^{d}$ & $H_{3}^{u}, H_{3}^{d}$ & $S$ & $T$ & $Y$ \\
\hline$Q_{6}$ & $\mathbf{2}_{1}$ & $\mathbf{1}_{+, 2}$ & $\mathbf{2}_{2}$ & $\mathbf{1}_{-, 1}$ & $\mathbf{2}_{2}$ & $\mathbf{1}_{+, 0}$ & $\mathbf{2}_{2}$ & $\mathbf{1}_{+, 0}$ & $\mathbf{1}_{-, 3}$ & $\mathbf{2}_{2}$ & $\mathbf{1}_{-, 1}$ & $\mathbf{2}_{1}$ & $\mathbf{2}_{2}$ & $\mathbf{1}_{+, 2}$ \\
\hline$Z_{4}$ & 3 & 3 & 0 & 0 & 1 & 1 & 2 & 2 & 2 & 1 & 1 & 2 & 2 & 0 \\
\hline$R$ & - & - & - & - & - & - & - & - & - & + & + & + & + & + \\
\hline
\end{tabular}

TABLE I: $\quad Q_{6} \times Z_{4} \times R$ assignment of the chiral supermultiplets, where $R$ is the $R$ parity. This is an alternative assignment to the one given in $[26]$. The abelian $Z_{4}$ is also an alterative to the abelian discrete $R$ symmetry $Z_{12 R}$ [26], for which one needs more singlets to construct a desired Higgs sector. The anomalies, $Q_{6}\left[S U(2)_{L}\right]^{2}, Q_{6}\left[S U(3)_{C}\right]^{2}, Z_{4}\left[S U(2)_{L}\right]^{2}$ and $Z_{4}\left[S U(3)_{C}\right]^{2}$, can be cancelled by the GreenSchwarz mechanism if, for instance, $\kappa_{2}=\kappa_{3}$ is satisfied [38], where $\kappa_{2}$ and $\kappa_{3}$ are the Kac-Moody levels for $S U(2)_{L}$ and $S U(3)_{C}$, respectively.

where

$$
\begin{aligned}
W_{Q}= & \sum_{I, i, j, k=1,2,3}\left(Y_{i j}^{u I} Q_{i} U_{j}^{c} H_{I}^{u}+Y_{i j}^{d I} Q_{i} D_{j}^{c} H_{I}^{d}\right) \\
W_{L}= & \sum_{I, i, j, k=1,2,3}\left(Y_{i j}^{e I} L_{i} E_{j}^{c} H_{I}^{d}+Y_{i j}^{\nu I} L_{i} N_{j}^{c} H_{I}^{u}\right)+\frac{m_{N}}{2} \sum_{i=1,2}\left(N_{i}^{c}\right)^{2}+\frac{\lambda_{N}}{2}\left(N_{3}^{c}\right)^{2} Y, \\
W_{H}= & m_{T}\left(T_{1}^{2}+T_{2}^{2}\right)+m_{Y} Y^{2}+\lambda_{S}\left(S_{1}^{2}+S_{2}^{2}\right) Y \\
& +\lambda_{1}\left(H_{1}^{u} S_{2}+H_{2}^{u} S_{1}\right) H_{3}^{d}+\lambda_{2}\left(H_{1}^{d} S_{2}+H_{2}^{d} S_{1}\right) H_{3}^{u} \\
& +\lambda_{3}\left[-\left(H_{1}^{u} H_{1}^{d}-H_{2}^{u} H_{2}^{d}\right) T_{1}+\left(H_{1}^{u} H_{2}^{d}+H_{2}^{u} H_{1}^{d}\right) T_{2}\right] .
\end{aligned}
$$

The Yukawa matrices $Y$ 's are given by

$$
\begin{aligned}
& \mathbf{Y}^{u 1(d 1)}=\left(\begin{array}{ccc}
0 & 0 & 0 \\
0 & 0 & Y_{b}^{u(d)} \\
0 & Y_{b^{\prime}}^{u(d)} & 0
\end{array}\right), \mathbf{Y}^{u 2(d 2)}=\left(\begin{array}{ccc}
0 & 0 & Y_{b}^{u(d)} \\
0 & 0 & 0 \\
-Y_{b^{\prime}}^{u(d)} & 0 & 0
\end{array}\right) \\
& \mathbf{Y}^{u 3(d 3)}=\left(\begin{array}{ccc}
0 & Y_{c}^{u(d)} & 0 \\
Y_{c}^{u(d)} & 0 & 0 \\
0 & 0 & Y_{a}^{u(d)}
\end{array}\right) \\
& \mathbf{Y}^{e 1}=\left(\begin{array}{ccc}
-Y_{c}^{e} & 0 & Y_{b}^{e} \\
0 & Y_{c}^{e} & 0 \\
Y_{b^{\prime}}^{e} & 0 & 0
\end{array}\right), \mathbf{Y}^{e 2}=\left(\begin{array}{ccc}
0 & Y_{c}^{e} & 0 \\
Y_{c}^{e} & 0 & Y_{b}^{e} \\
0 & Y_{b^{\prime}}^{e} & 0
\end{array}\right), \mathbf{Y}^{e 3}=0 \\
& \mathbf{Y}^{\nu 1}=\left(\begin{array}{ccc}
-Y_{c}^{\nu} & 0 & 0 \\
0 & Y_{c}^{\nu} & 0 \\
Y_{b^{\prime}}^{\nu} & 0 & 0
\end{array}\right), \mathbf{Y}^{\nu 2}=\left(\begin{array}{ccc}
0 & Y_{c}^{\nu} & 0 \\
Y_{c}^{\nu} & & 0 \\
0 & Y_{b^{\prime}}^{\nu} & 0
\end{array}\right) \\
& \mathbf{Y}^{\nu 3}=\left(\begin{array}{ccc}
0 & 0 & 0 \\
0 & 0 & 0 \\
0 & 0 & Y_{a}^{\nu}
\end{array}\right) \text {. }
\end{aligned}
$$


All the parameters appearing above are real, because we assume a spontaneous CP violation to occur. We will shortly come back to this issue.

The $Z_{4}$ charges of the fields are so chosen that the most general $Q_{6} \times Z_{4} \times R$ invariant Higgs superpotential (10) has an accidental symmetry:

$$
H_{1}^{u, d} \leftrightarrow H_{2}^{u, d}, S_{1} \leftrightarrow S_{2}, T_{1} \rightarrow-T_{1},
$$

where $H_{3}^{u, d}, T_{2}$ and $Y$ do not transform. This symmetry ensures the stability of the VEV structure

$$
\begin{gathered}
\left\langle H_{1}^{u}\right\rangle=\left\langle H_{2}^{u}\right\rangle=\frac{1}{2} v_{D}^{u} e^{i \theta^{u}},\left\langle H_{1}^{d}\right\rangle=\left\langle H_{2}^{d}\right\rangle=\frac{1}{2} v_{D}^{d} e^{i \theta^{d}},\left\langle H_{3}^{u, d}\right\rangle=\frac{1}{\sqrt{2}} v_{3}^{u, d} e^{i \theta_{3}^{u, d}}, \\
\left\langle S_{1}\right\rangle=\left\langle S_{2}\right\rangle=v^{S} e^{i\left(\theta^{S} / 2\right)},\left\langle T_{2}\right\rangle=v^{T} e^{i\left(\theta^{T} / 2\right)},\langle Y\rangle=v^{Y} e^{i\left(\theta^{Y} / 2\right)},\left\langle T_{1}\right\rangle=0,
\end{gathered}
$$

where $v$ 's are non-vanishing real quantities.

The total Higgs potential consists of the supersymmetric part ( $D$ terms and $F$ terms) and the $Q_{6} \times Z_{4} \times R$ invariant SSB part, where the $B$ and $A$ terms are given by

$$
\begin{aligned}
\mathcal{L}_{\mathrm{SSB}}^{H}= & B_{T}\left(\tilde{T}_{1}^{2}+\tilde{T}_{2}^{2}\right)+B_{Y} \tilde{Y}^{2}+\lambda_{S} A_{S}\left(\tilde{S}_{1}^{2}+\tilde{S}_{2}^{2}\right) \tilde{Y} \\
& +\lambda_{1} A_{1}\left(\tilde{H}_{1}^{u} \tilde{S}_{2}+\tilde{H}_{2}^{u} \tilde{S}_{1}\right) \tilde{H}_{3}^{d}+\lambda_{2} A_{2}\left(\tilde{H}_{1}^{d} \tilde{S}_{2}+\tilde{H}_{2}^{d} \tilde{S}_{1}\right) \tilde{H}_{3}^{u} \\
& +\lambda_{3} A_{3}\left[-\left(\tilde{H}_{1}^{u} \tilde{H}_{1}^{d}-\tilde{H}_{2}^{u} \tilde{H}_{2}^{d}\right) \tilde{T}_{1}+\left(\tilde{H}_{1}^{u} \tilde{H}_{2}^{d}+\tilde{H}_{2}^{u} \tilde{H}_{1}^{d}\right) \tilde{T}_{2}\right]+\text { h.c. }
\end{aligned}
$$

(The fields with a tilde are the bosonic components of the corresponding supermultiplets.) As in the case of the supersymmetric part, $B$ 's and $A$ 's are assumed to be real. We have investigated the minimization conditions for the angles $\theta$ 's given in (15), and found that a nontrivial solution of the conditions can exist. How the mass spectrum for this nontrivial solution looks like is still an open problem. A complete analysis of this problem, which is similar to that of [39], will go beyond the scope of the present paper. We will publish the analysis elsewhere. To proceed, we here simply assume that there exist a nontrivial CP violating set of VEV's.

\section{Fermion mass matrices and diagonalization}

We assume that VEVs take the form (15), from which we obtain the fermion mass matrices. 


\section{Quark sector}

The quark mass matrices are given by

$$
\begin{aligned}
\mathbf{m}^{u} & =\frac{1}{2}\left(\begin{array}{ccc}
0 & \sqrt{2} Y_{c}^{u} v_{3}^{u} e^{-i \theta_{3}^{u}} & Y_{b}^{u} v_{D}^{u} e^{-i \theta^{u}} \\
\sqrt{2} Y_{c}^{u} v_{3}^{u} e^{-i \theta_{3}^{u}} & 0 & Y_{b}^{u} v_{D}^{u} e^{-i \theta^{u}} \\
-Y_{b^{\prime}}^{u} v_{D}^{u} e^{-i \theta^{u}} & Y_{b^{\prime}}^{u} v_{D}^{u} e^{-i \theta^{u}} & \sqrt{2} Y_{a}^{u} v_{3}^{u} e^{-i \theta_{3}^{u}}
\end{array}\right), \\
\mathbf{m}^{d} & =\frac{1}{2}\left(\begin{array}{ccc}
0 & \sqrt{2} Y_{c}^{d} v_{3}^{d} e^{-i \theta_{3}^{d}} & Y_{b}^{d} v_{D}^{d} e^{-i \theta^{d}} \\
\sqrt{2} Y_{c}^{d} v_{3}^{d} e^{-i \theta_{3}^{d}} & 0 & Y_{b}^{d} v_{D}^{d} e^{-i \theta^{d}} \\
-Y_{b^{\prime}}^{d} v_{D}^{d} e^{-i \theta^{d}} & Y_{b^{\prime}}^{d} v_{D}^{d} e^{-i \theta^{d}} & \sqrt{2} Y_{a}^{d} v_{3}^{d} e^{-i \theta_{3}^{d}}
\end{array}\right) .
\end{aligned}
$$

In the present case, the unitary matrices that rotate quarks have the following form: $U_{u L(R)}=$ $R_{L(R)} P_{L(R)}^{u} O_{L(R)}^{u}$, where $O^{\prime}$ 's are orthogonal matrices, and

$$
\begin{aligned}
R_{L} & =\frac{1}{\sqrt{2}}\left(\begin{array}{ccc}
1 & 1 & 0 \\
-1 & 1 & 0 \\
0 & 0 & \sqrt{2}
\end{array}\right), R_{R}=\frac{1}{\sqrt{2}}\left(\begin{array}{ccc}
-1 & -1 & 0 \\
-1 & 1 & 0 \\
0 & 0 & \sqrt{2}
\end{array}\right) \\
P_{L}^{u} & =\left(\begin{array}{ccc}
1 & 0 & 0 \\
0 & \exp \left(i 2 \Delta \theta^{u}\right) & 0 \\
0 & 0 & \exp \left(i \Delta \theta^{u}\right)
\end{array}\right) \\
P_{R}^{u} & =\left(\begin{array}{ccc}
\exp \left(i 2 \Delta \theta^{u}\right) & 0 & 0 \\
0 & 1 & 0 \\
0 & 0 & \exp \left(i \Delta \theta^{u}\right)
\end{array}\right) \exp \left(i \theta_{3}^{u}\right), \\
\Delta \theta^{u} & =\theta_{3}^{u}-\theta^{u},
\end{aligned}
$$

and similarly for the down sector. The phase matrices $P_{L, R}^{u, d}$ can rotate away the phases of the mass matrices, so that we can bring $\mathbf{m}^{u}$ into a real form

$$
\hat{\mathbf{m}}^{u}=P_{L}^{u \dagger} R_{L}^{T} \mathbf{m}^{u} R_{R} P_{R}^{u}=m_{t}\left(\begin{array}{ccc}
0 & q_{u} / y_{u} & 0 \\
-q_{u} / y_{u} & 0 & b_{u} \\
0 & b_{u}^{\prime} & y_{u}^{2}
\end{array}\right),
$$

which can be then diagonalized as ${ }^{3}$

$$
O_{L}^{u T} \hat{\mathbf{m}}^{u} O_{R}^{u}=\left(\begin{array}{ccc}
m_{u} & 0 & \\
0 & m_{c} & 0 \\
0 & 0 & m_{t}
\end{array}\right)
$$

and similarly for $\mathbf{m}^{d}$.

The CKM matrix $V_{\mathrm{CKM}}$ is given by

$$
V_{\mathrm{CKM}}=O_{L}^{u T} P_{L}^{u \dagger} P_{L}^{d} O_{L}^{d}
$$

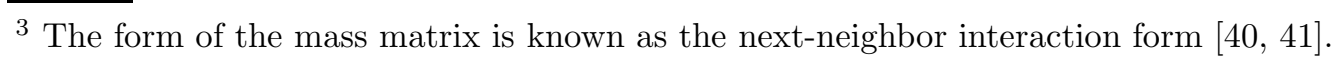


and for the set of the parameters

$$
\begin{aligned}
& \theta_{q}=\theta_{3}^{d}-\theta^{d}-\theta_{3}^{u}+\theta^{u}=-1.25, q_{u}=0.0002143, b_{u}=0.04443, b_{u}^{\prime}=0.09338 \\
& y_{u}=0.99732, q_{d}=0.005091, b_{d}=0.02570, b_{d}^{\prime}=0.77606, y_{d}=0.7940
\end{aligned}
$$

we obtain

$$
\begin{aligned}
m_{u} / m_{t} & =1.10 \times 10^{-5}, m_{c} / m_{t}=4.16 \times 10^{-3}, m_{d} / m_{b}=1.22 \times 10^{-3}, m_{s} / m_{b}=2.13 \times 10^{-2}, \\
\left|V_{\mathrm{CKM}}\right| & =\left(\begin{array}{lll}
0.9747 & 0.2236 & 0.0040 \\
0.2234 & 0.9738 & 0.0421 \\
0.0093 & 0.0413 & 0.9991
\end{array}\right), \sin 2 \beta\left(\phi_{1}\right)=0.738
\end{aligned}
$$

The experimental values to be compared are [42]:

$$
\begin{aligned}
\left|V_{\mathrm{CKM}}^{\exp }\right| & =\left(\begin{array}{ccc}
0.9739 \text { to } 0.9751 & 0.221 \text { to } 0.227 & 0.0029 \text { to } 0.0045 \\
0.221 \text { to } 0.227 & 0.9730 \text { to } 0.9744 & 0.039 \text { to } 0.044 \\
0.0048 \text { to } 0.014 & 0.037 \text { to } 0.043 & 0.9990 \text { to } 0.9992
\end{array}\right), \\
\sin 2 \beta\left(\phi_{1}\right) & =0.736 \pm 0.049 .
\end{aligned}
$$

The quark masses at $M_{Z}$ are given by [43]

$$
\begin{aligned}
m_{u} / m_{d} & =0.541 \pm 0.086(0.61), m_{s} / m_{d}=18.9 \pm 1.6(17.5) \\
m_{c} & =0.73 \pm 0.17(0.72) \mathrm{GeV}, m_{s}=0.058 \pm 0.015(0.062) \mathrm{GeV} \\
m_{t} & =175 \pm 6 \mathrm{GeV}, m_{b}=2.91 \pm 0.07 \mathrm{GeV}
\end{aligned}
$$

where the values in the parentheses are the theoretical values obtained from (27) for $m_{t}=174$ $\mathrm{GeV}$ and $m_{b}=2.9 \mathrm{GeV}$. So, we see that the model can well reproduce the experimentally measured parameters. Because of the family symmetry, the CKM parameters and the quark masses are related. In Fig. 1 we plot the predicted area in the $\sin 2 \phi_{1}-\phi_{3}$ plane. We see

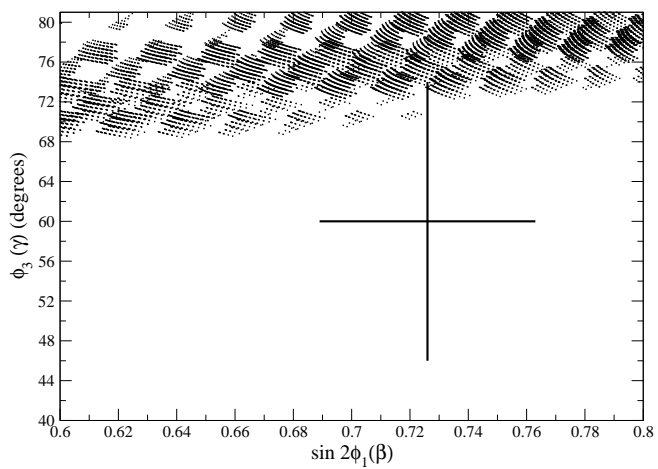

FIG. 1: Predicted area in the $\sin 2 \phi_{1}-\phi_{3}$ plane. The vertical and horizontal lines correspond to the experimental values, $\sin 2 \phi_{1}(\beta)=0.726 \pm 0.037$ and $\phi_{3}=\left(60^{\circ} \pm 14^{\circ}\right)$ [42, 444]. 
from Fig. 1 that the model requires

$$
\phi_{3} \gtrsim 68^{\circ} .
$$

Another quantity to be compared may be $\left|V_{t d} / V_{t s}\right|$, whose experimental value has been recently obtained from the observation of $b \rightarrow d+\gamma$ in the $B$ decays [45]:

$$
\begin{aligned}
\text { Model : }\left|V_{t d} / V_{t s}\right| & =0.21-0.23 \\
\text { Exp. : }\left|V_{t d} / V_{t s}\right|=0.200 & { }_{-0.025}^{+0.026}\left(\text { exp. }{ }_{-0.029}^{+0.038}\right. \text { (theo.). }
\end{aligned}
$$

We may conclude that 9 independent parameters of the model can well describe 10 physical observables.

The mass matrices given in the present model can be analytically diagonalized [46], and the approximate formula for $V_{\mathrm{CKM}}$ implies that

$$
\begin{aligned}
V_{u s} & \simeq 0.794 \sqrt{\frac{m_{d}}{m_{s}}}-\sqrt{\frac{m_{u}}{m_{c}}} e^{-i 2.5}, V_{c d} \simeq-\sqrt{\frac{m_{u}}{m_{c}}}+0.794 \sqrt{\frac{m_{d}}{m_{s}}} e^{-i 2.5}, \\
V_{c b} & \simeq 0.81\left(\frac{m_{s}}{m_{b}}\right) e^{-i 2.5}-9.63\left(\frac{m_{c}}{m_{t}}\right) e^{-i 1.25},
\end{aligned}
$$

which should be compared with the Fritzsch formulas [1]].

Finally, we give the unitary matrices that rotate the quarks for the choice of the parameters given in (26):

$$
U_{u L(R)}=R_{L(R)}^{u} P_{L(R)}^{u} O_{L(R)}^{u}, U_{d L(R)}=R_{L(R)}^{d} P_{L(R)}^{d} O_{L(R)}^{d},
$$

where $R_{L, R}^{u}$ and $R_{L, R}^{d}$ are given in (19) and (201), and

$$
\begin{aligned}
& O_{L}^{u} \simeq\left(\begin{array}{ccc}
0.9987 & 0.0514 & 2 \times 10^{-5} \\
0.0513 & -0.9977 & 0.0442 \\
-0.0023 & 0.0442 & 0.9990
\end{array}\right), O_{R}^{u} \simeq\left(\begin{array}{ccc}
-0.9987 & 0.0515 & -10^{-5} \\
0.0513 & 0.9943 & 0.0933 \\
-0.0048 & -0.0932 & 0.9956
\end{array}\right), \\
& O_{L}^{d} \simeq\left(\begin{array}{ccc}
0.9834 & 0.1814 & 0.0050 \\
0.1813 & -0.9833 & 0.0162 \\
-0.0078 & 0.0150 & 0.9999
\end{array}\right), O_{R}^{d} \simeq\left(\begin{array}{ccc}
-0.9552 & 0.2960 & -0.0001 \\
0.1866 & 0.6025 & 0.7760 \\
-0.2298 & -0.7412 & 0.6308
\end{array}\right) .
\end{aligned}
$$

Using these orthogonal matrices and the matrices defined in (19) (22), Eq. (33) gives the 
unitary matrices in the explicit form:

$$
\begin{aligned}
& U_{u L}=\left(\begin{array}{ccc}
0.706 & 0.0363 & 1.42 \times 10^{-5} \\
-0.706 & -0.0363 & -1.42 \times 10^{-5} \\
0 & 0 & 0
\end{array}\right) \\
& +e^{2 i \Delta \theta^{u}}\left(\begin{array}{ccc}
0.0363 & -0.705 & 0.0313 \\
0.0363 & -0.705 & 0.0313 \\
-0.00229 e^{-i \Delta \theta^{u}} & 0.0442 e^{-i \Delta \theta^{u}} & 0.999 e^{-i \Delta \theta^{u}}
\end{array}\right), \\
& U_{d L}=\left(\begin{array}{ccc}
0.695 & 0.128 & 0.00352 \\
-0.695 & -0.128 & -0.00352 \\
0 & 0 & 0
\end{array}\right) \\
& +e^{2 i \Delta \theta^{d}}\left(\begin{array}{ccc}
0.128 & -0.695 & 0.0115 \\
0.128 & -0.695 & 0.0115 \\
-0.00783 e^{-i \Delta \theta^{d}} & 0.0150 e^{-i \Delta \theta^{d}} & 1.00 e^{-i \Delta \theta^{d}}
\end{array}\right), \\
& U_{u R}=e^{i \theta_{3}^{u}}\left(\begin{array}{ccc}
0.0363 & 0.703 & 0.0660 \\
0.0363 & 0.703 & 0.0660 \\
0 & 0 & 0
\end{array}\right) \\
& +e^{2 i \Delta \theta^{u}+i \theta_{3}^{u}}\left(\begin{array}{ccc}
-0.706 & 0.0364 & -6.72 \times 10^{-6} \\
0.706 & -0.0364 & 6.72 \times 10^{-6} \\
-0.00482 e^{-i \Delta \theta^{u}} & -0.0932 e^{-i \Delta \theta^{u}} & 0.996 e^{-i \Delta \theta^{u}}
\end{array}\right) \text {, } \\
& U_{d R}=e^{i \theta_{3}^{d}}\left(\begin{array}{ccc}
0.132 & 0.426 & 0.549 \\
0.132 & 0.426 & 0.549 \\
0 & 0 & 0
\end{array}\right) \\
& +e^{2 i \Delta \theta^{d}+i \theta_{3}^{d}}\left(\begin{array}{ccc}
-0.675 & 0.209 & -7.35 \times 10^{-5} \\
0.675 & -0.209 & 7.35 \times 10^{-5} \\
-0.230 e^{-i \Delta \theta^{d}} & -0.741 e^{-i \Delta \theta^{d}} & 0.631 e^{-i \Delta \theta^{d}}
\end{array}\right) .
\end{aligned}
$$

The unitary matrices above will be used when discussing the SUSY flavor problem in sect. III and proton decay in sect. IV.

\section{Lepton sector}

The mass matrices in the lepton sectors are:

$$
\begin{aligned}
\mathbf{m}^{e} & =\frac{1}{2}\left(\begin{array}{ccc}
-Y_{c}^{e} & Y_{c}^{e} & Y_{b}^{e} \\
Y_{c}^{e} & Y_{c}^{e} & Y_{b}^{e} \\
Y_{b^{\prime}}^{e} & Y_{b^{\prime}}^{e} & 0
\end{array}\right) v_{D}^{d} e^{-i \theta^{d}}, \\
\mathbf{m}^{\nu} & =\frac{1}{2}\left(\begin{array}{ccc}
-Y_{c}^{\nu} & Y_{c}^{\nu} & 0 \\
Y_{c}^{\nu} & Y_{c}^{\nu} & 0 \\
Y_{b^{\prime}}^{\nu} & Y_{b^{\prime}}^{\nu} & \sqrt{2} Y_{a}^{\nu} \tan \gamma^{u} e^{i\left(\theta_{3}^{u}-\theta^{u}\right)}
\end{array}\right) v_{D}^{u} e^{i \theta^{u}}
\end{aligned}
$$


where $\tan \gamma^{u}=v_{3}^{u} / v_{D}^{u}$. We start with the mass matrix of the charged leptons $\mathbf{m}^{e}$ :

$$
U_{e L}^{\dagger} \mathbf{m}^{e} U_{e R}=\left(\begin{array}{ccc}
m_{e} & 0 & \\
0 & m_{\mu} & 0 \\
0 & 0 & m_{\tau}
\end{array}\right)
$$

One finds [? ] that $U_{e L}$ and $U_{e R}$ can be approximately written as

$$
\begin{gathered}
U_{e L}=R\left(\begin{array}{ccc}
-\epsilon_{e}\left(1+\epsilon_{\mu}^{2}\right) & -(1 / \sqrt{2})\left(1-\epsilon_{e}^{2}-\epsilon_{e}^{2} \epsilon_{\mu}^{2}\right) & 1 / \sqrt{2} \\
\epsilon_{e}\left(1-\epsilon_{\mu}^{2}\right) & (1 / \sqrt{2})\left(1-\epsilon_{e}^{2}+\epsilon_{e}^{2} \epsilon_{\mu}^{2}\right) & 1 / \sqrt{2} \\
1-\epsilon_{e}^{2} & -\sqrt{2} \epsilon_{e} & \sqrt{2} \epsilon_{e} \epsilon_{\mu}^{2}
\end{array}\right), \\
U_{e R}=R\left(\begin{array}{ccc}
1-\epsilon_{\mu}^{2} / 2 & -\epsilon_{e}^{2}\left(1-\epsilon_{\mu}^{2}\right) & \epsilon_{\mu} \\
-\epsilon_{e}^{2}\left(1-\epsilon_{\mu}^{2} / 2\right) & -1 & 0 \\
-\epsilon_{\mu} & \epsilon_{e}^{2} \epsilon_{\mu} & 1-\epsilon_{\mu}^{2} / 2
\end{array}\right) e^{i \theta^{d}},
\end{gathered}
$$

where $R$ interchanges the first and second row,

$$
R=\left(\begin{array}{lll}
0 & 1 & 0 \\
1 & 0 & 0 \\
0 & 0 & 1
\end{array}\right)
$$

and $\epsilon_{\mu}=m_{\mu} / m_{\tau}$ and $\epsilon_{e}=m_{e} /\left(\sqrt{2} m_{\mu}\right)$. In the limit $m_{e}=0$, the unitary matrix $U_{e L}$ becomes

$$
\left(\begin{array}{ccc}
0 & 1 / \sqrt{2} & 1 / \sqrt{2} \\
0 & -1 / \sqrt{2} & 1 / \sqrt{2} \\
1 & 0 & 0
\end{array}\right)
$$

which is the origin for a maximal mixing of the atmospheric neutrinos.

As for the neutrino masses, we assume that a see-saw mechanism [47] takes place. As we can see from (9), the mass matrix for the right-handed neutrinos is diagonal: $\mathbf{m}_{N}=$ $\left((-1)^{\eta} m_{N},(-1)^{\eta} m_{N}, \lambda_{N} v^{Y} \exp \left(i \theta_{Y} / 2\right)\right), \quad(\eta=0$ or 1$)$, where $m_{N}$ and $\lambda_{N} v^{Y}$ are real and positive by assumption. Therefore, the Majorana mass matrix for the left-handed neutrinos becomes

$$
\begin{aligned}
\mathbf{M}_{\nu} & =\mathbf{m}^{\nu} \mathbf{m}_{N}^{-1}\left(\mathbf{m}^{\nu}\right)^{T} \\
& =(-1)^{\eta} e^{i 2 \theta^{u}} R\left(\begin{array}{ccc}
2\left(\rho_{2}\right)^{2} & 0 & 2 \rho_{2} \rho_{4} \\
0 & 2\left(\rho_{2}\right)^{2} & 0 \\
2 \rho_{2} \rho_{4} & 0 & 2\left(\rho_{4}\right)^{2}+\left(\rho_{3}\right)^{2} \exp i 2 \varphi_{3}
\end{array}\right) R,
\end{aligned}
$$

where $R$ is given in (45), and

$$
\begin{aligned}
2 \varphi_{3} & =2\left(\theta_{3}^{u}-\theta^{u}\right)+\eta \pi-\theta^{Y} / 2 \\
\rho_{2} & =\frac{1}{2} Y_{c}^{\nu} v_{D}^{u} / \sqrt{m_{N}}, \rho_{4}=\frac{1}{2} Y_{b^{\prime}}^{\nu} v_{D}^{u} / \sqrt{m_{N}}, \rho_{3}=\frac{1}{\sqrt{2}} Y_{a}^{\nu} v_{3}^{u} / \sqrt{Y_{N} v^{Y}} .
\end{aligned}
$$


The factor $(-1)^{\eta} e^{i 2 \theta^{u}}$ has no effect, and so we ignore it in the following discussions.

Noticing that the $\rho$ 's in (46) are real numbers, we recall that that $\mathbf{M}_{\nu}$ can be diagonalized as 34 ]

$$
U_{\nu}^{T} \mathbf{M}_{\nu} U_{\nu}=\left(\begin{array}{ccc}
m_{\nu_{1}} & 0 & 0 \\
0 & m_{\nu_{2}} & 0 \\
0 & 0 & m_{\nu_{3}}
\end{array}\right)
$$

where $c_{12}=\cos \theta_{12}$, and $s_{12}=\sin \theta_{12}$, and

$$
\begin{aligned}
U_{\nu} & =R\left(\begin{array}{ccc}
-s_{12} e^{i\left(\phi_{\nu}-i \varphi_{1}\right) / 2} & c_{12} e^{i\left(\phi_{\nu}-i \varphi_{2}\right) / 2} & 0 \\
0 & 0 & -1 \\
c_{12} e^{-i\left(\phi_{\nu}+i \varphi_{1}\right) / 2} & s_{12} e^{-i\left(\phi_{\nu}+i \varphi_{2}\right) / 2} & 0
\end{array}\right), \\
m_{\nu_{3}} \sin \phi_{\nu} & =m_{\nu_{2}} \sin \varphi_{2}=m_{\nu_{1}} \sin \varphi_{1}, 2 \varphi_{3}=\varphi_{1}+\varphi_{2}, \\
\frac{m_{\nu_{2}}^{2}}{\Delta m_{23}^{2}} & \simeq \frac{1}{\sin ^{2} 2 \theta_{12} \cos ^{2} \phi_{\nu}}-\tan ^{2} \phi_{\nu} \text { for }|r|<<1 .
\end{aligned}
$$

It turns out that only an inverted mass spectrum

$$
m_{\nu_{3}}<m_{\nu_{1}}, m_{\nu_{2}}
$$

is consistent with the experimental constraint $\left|\Delta m_{21}^{2}\right|<\left|\Delta m_{23}^{2}\right|$ in the present model. Note that Eq. (51) is satisfied for

$$
2 \varphi_{3}=\varphi_{1}+\varphi_{2} \sim \pm \pi
$$

and not for $\varphi_{1} \sim \varphi_{2}$.

The mixing matrix $V_{\text {MNS }}$ can be obtained from $U_{e L}^{\dagger} U_{\nu}$, where $U_{e L}$ and $U_{\nu}$ are given in (43) and (150), respectively. The product $U_{e L}^{\dagger} U_{\nu}$ can be brought by an appropriate phase transformation to a popular form, which in the present model approximately assumes the form

$$
V_{\mathrm{MNS}} \simeq\left(\begin{array}{ccc}
c_{12} & s_{12} & s_{13} e^{-i \delta} \\
-s_{12} / \sqrt{2} & c_{12} / \sqrt{2} & 1 / \sqrt{2} \\
s_{12} / \sqrt{2} & -c_{12} / \sqrt{2} & 1 / \sqrt{2}
\end{array}\right) \times\left(\begin{array}{ccc}
1 & 0 & 0 \\
0 & e^{i \alpha} & 0 \\
0 & 0 & e^{i \beta}
\end{array}\right)
$$

with

$$
\begin{aligned}
s_{13} & \simeq \epsilon_{e}=\frac{m_{e}}{\sqrt{2} m_{\mu}}=3.4 \cdots \times 10^{-3}, \delta_{C P} \simeq-\phi_{\nu} \\
\sin 2 \alpha & =\sin \left(\varphi_{1}-\varphi_{2}\right), \quad \sin 2 \beta=\sin \left(\varphi_{1}-\varphi_{\nu}\right),
\end{aligned}
$$

where $\varphi_{1}, \varphi_{2}$ and $\phi_{\nu}$ are defined in (51) ${ }^{4}$. There are seven independent parameters to describe 12 parameters $(3+3=6$ masses, three angles and three phases $)$ of the lepton

\footnotetext{
${ }^{4}$ Unfortunately, this value of $s_{13}$ is too small to be measured [48].
} 
sector. Therefore, the effective Majorana mass $<m_{e e}>$ in neutrinoless double $\beta$ decay, for instance, can be predicted. In Fig. 2 we plot $\left\langle m_{e e}>\right.$ as a function of $\sin \phi_{\nu}$ for $\sin ^{2} \theta_{12}=0.3, \Delta m_{21}^{2}=6.9 \times 10^{-5} \mathrm{eV}^{2}$ and $\Delta m_{23}^{2}=1.4,2.3,3.0 \times 10^{-3} \mathrm{eV}^{2}$ [49]. As we see from Fig. 2, the prediction is consistent with recent experiments [50, 51].

We will use the unitary matrices given in (43), (44) and (150) in sect. III and IV, and will see that the small parameter $s_{13}=\epsilon_{e}=m_{e} /(\sqrt{2}) / m_{\mu} \simeq 0.0034$ appears as a suppression factor in FCNCs as well as in some of proton decay modes.

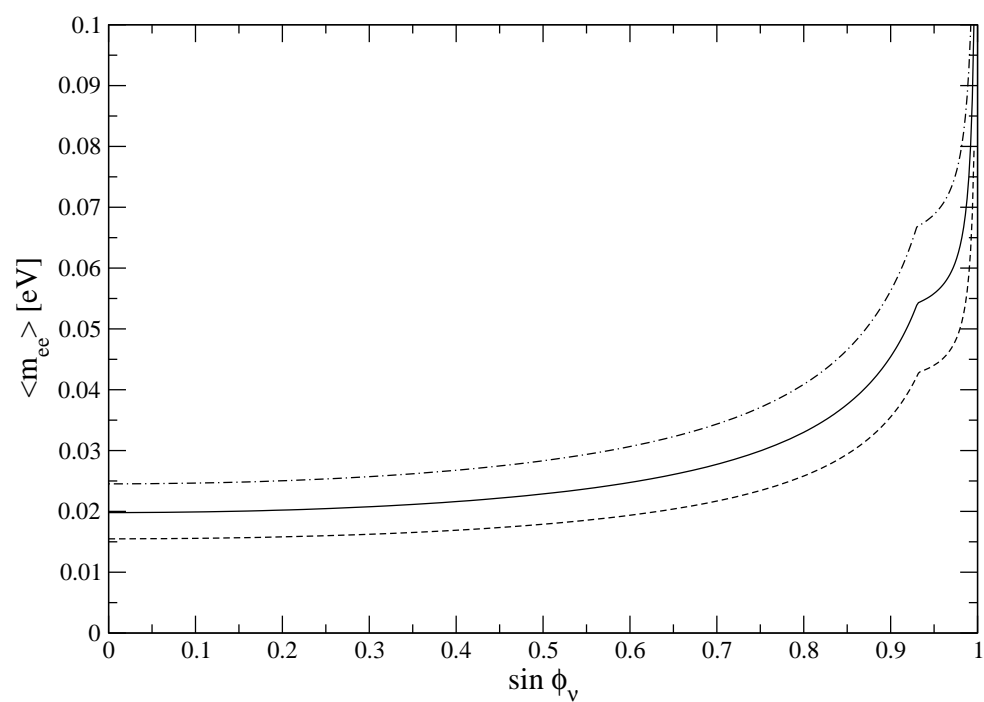

FIG. 2: The effective Majorana mass $<m_{e e}>$ as a function of $\sin \phi_{\nu}$ with $\sin ^{2} \theta_{12}=0.3$ and $\Delta m_{21}^{2}=6.9 \times 10^{-5} \mathrm{eV}^{2}$. The dashed, solid and dot-dashed lines stand for $\Delta m_{23}^{2}=1.4,2.3$ and $3.0 \times 10^{-3} \mathrm{eV}^{2}$, respectively. The $\Delta m_{21}^{2}$ dependence is very small.

\section{THE SUSY FLAVOR PROBLEM}

If three generations of a family is put into a one-dimensional and two-dimensional irreps of any dihedral group, then the soft scalar mass matrix for the sfermions has always a diagonal form:

$$
\tilde{\mathbf{m}}_{(q, \ell) L L}^{2}=m_{\tilde{q}, \tilde{\ell}}^{2}\left(\begin{array}{ccc}
a_{L}^{q, \ell} & 0 & 0 \\
0 & a_{L}^{q, \ell} & 0 \\
0 & 0 & b_{L}^{q, \ell}
\end{array}\right), \tilde{\mathbf{m}}_{a R R}^{2}=m_{\tilde{q}, \tilde{\ell}}^{2}\left(\begin{array}{ccc}
a_{R}^{a} & 0 & 0 \\
0 & a_{R}^{a} & 0 \\
0 & 0 & b_{R}^{a}
\end{array}\right) \quad(a=u, d, e),
$$

where $m_{\tilde{q}, \tilde{\ell}}$ denote the average of the squark and slepton masses, respectively, and $\left(a_{L(R)}, b_{L(R)}\right)$ are dimensionless free real parameters of $O(1)$. Further, since the trilinear interactions ( $A$ terms) are also $Q_{6}$ invariant, the left-right mass matrices assume the form

$$
\left(\tilde{\mathbf{m}}_{a L R}^{2}\right)_{i j}=A_{i j}^{a}\left(\mathbf{m}^{a}\right)_{i j} \quad(a=u, d, e),
$$


where $A_{i}^{a}$ 's are free parameters of dimension one, and the fermion masses $\mathbf{m}$ 's are given in (17), (18) and (40). Here we assume that $A_{i}^{a}$ 's are in the same order as the gaugino masses. They are real, because we impose CP invariance at the Lagrangian level.

We work in the super CKM basis and calculate

$$
\Delta_{L L}^{a}=U_{a L}^{\dagger} \tilde{\mathbf{m}}_{(q, \ell) L L}^{2} U_{a L} \text { and } \Delta_{R R(L R)}^{a}=U_{a R(L)}^{\dagger} \tilde{\mathbf{m}}_{a R R(L R)}^{2} U_{a R}
$$

to parameterize FCNCs and CP violations coming from the SSB sector, where the unitary matrices $U$ 's are given in (33), (43) and (44). In doing so, one observes that something interesting happens; a phase alignment. This is because the only source for CP phases comes from VEVs (15). To see the phase alignment, we first observe that the matrices $R_{L, R}$ and the phase rotation matrices $P_{L, R}^{u, d}$, given in (19), (20) and (21), commute with the scalar soft mass matrices $\tilde{\mathbf{m}}_{q L L}^{2}$ and $\tilde{\mathbf{m}}_{u, d R R}^{2}$. This implies that $\Delta_{L L, R R}^{u, d}$ are real, where $\Delta_{L L, R R}^{e}$ is trivially real as we see from (43) and (44). As for the left-right soft mass squared (59), we find that $\left(P_{L}^{a}\right)^{\dagger}\left(R_{L}^{a}\right)^{T} \tilde{\mathbf{m}}_{a L R}^{2} R_{R}^{a} P_{R}^{a}$ is a real matrix for all $a=u, d, e$. Consequently, no $C P$ violating processes induced by the SSB terms are possible in this model, satisfying the most stringent experimental constraint coming from the EDM of the neutron and the electron 10].

In [10], experimental bounds on the dimensionless quantities

$$
\delta_{L L, R R, L R}^{a}=\Delta_{L L, R R, L R}^{a} / m_{\tilde{q}, \tilde{\ell}}^{2} \quad(a=u, d, e)
$$

are given. The theoretical values of $\delta$ 's for the present model are calculated below, where

$$
\Delta a_{L}^{q, \ell}=a_{L}^{q, \ell}-b_{L}^{q, \ell}, \Delta a_{R}^{a}=a_{R}^{a}-b_{R}^{a}, \tilde{A}_{i}^{a}=\frac{A_{i}^{a}}{m_{\tilde{q}, \tilde{\ell}}} \quad(a=u, d, e)
$$

are introduced.

\section{$\underline{\text { Leptonic sector }(L L \text { and } R R) \text { : }}$}

$$
\begin{aligned}
& \left(\delta_{12}^{e}\right)_{L L}=\left(\delta_{21}^{e}\right)_{L L} \simeq 4.9 \times 10^{-3} \Delta a_{L}^{\ell} \\
& \left(\delta_{13}^{e}\right)_{L L}=\left(\delta_{31}^{e}\right)_{L L} \simeq-1.7 \times 10^{-5} \Delta a_{L}^{\ell} \\
& \left(\delta_{23}^{e}\right)_{L L}=\left(\delta_{32}^{e}\right)_{L L} \simeq 8.4 \times 10^{-8} \Delta a_{L}^{\ell} \\
& \left(\delta_{12}^{e}\right)_{R R}=\left(\delta_{21}^{e}\right)_{R R} \simeq 8.4 \times 10^{-8} \Delta a_{R}^{e} \\
& \left(\delta_{13}^{e}\right)_{R R}=\left(\delta_{31}^{e}\right)_{R R} \simeq 5.9 \times 10^{-2} \Delta a_{R}^{e} \\
& \left(\delta_{23}^{e}\right)_{R R}=\left(\delta_{32}^{e}\right)_{R R} \simeq-1.4 \times 10^{-6} \Delta a_{R}^{e} .
\end{aligned}
$$




\section{Leptonic sector $(L R)$ :}

$$
\begin{aligned}
& \left(\delta_{12}^{e}\right)_{L R} \simeq 5.1 \times 10^{-6}\left(\tilde{A}_{c}^{e}-\tilde{A}_{b^{\prime}}^{e}\right)\left(\frac{100 \mathrm{GeV}}{m_{\tilde{\ell}}}\right), \\
& \left(\delta_{21}^{e}\right)_{L R} \simeq 2.5 \times 10^{-8}\left(\tilde{A}_{c}^{e}-\tilde{A}_{b^{\prime}}^{e}\right)\left(\frac{100 \mathrm{GeV}}{m_{\tilde{\ell}}}\right), \\
& \left(\delta_{13}^{e}\right)_{L R} \simeq 3.1 \times 10^{-7}\left(\tilde{A}_{b^{\prime}}^{e}-\tilde{A}_{b}^{e}\right)\left(\frac{100 \mathrm{GeV}}{m_{\tilde{\ell}}}\right), \\
& \left(\delta_{31}^{e}\right)_{L R} \simeq 1.1 \times 10^{-3}\left(\tilde{A}_{c}^{e}-\tilde{A}_{b}^{e}\right)\left(\frac{100 \mathrm{GeV}}{m_{\tilde{\ell}}}\right), \\
& \left(\delta_{23}^{e}\right)_{L R} \simeq-1.5 \times 10^{-9}\left(\tilde{A}_{b^{\prime}}^{e}-\tilde{A}_{b}^{e}\right)\left(\frac{100 \mathrm{GeV}}{m_{\tilde{\ell}}}\right), \\
& \left(\delta_{32}^{e}\right)_{L R} \simeq-2.5 \times 10^{-8}\left(\tilde{A}_{c}^{e}-\tilde{A}_{b}^{e}\right)\left(\frac{100 \mathrm{GeV}}{m_{\tilde{\ell}}}\right) .
\end{aligned}
$$

$\underline{\text { Up quark sector }(L L \text { and } R R) \text { : }}$

$$
\begin{aligned}
& \left(\delta_{12}^{u}\right)_{L L}=\left(\delta_{21}^{u}\right)_{L L} \simeq 1.0 \times 10^{-4} \Delta a_{L}^{q}, \\
& \left(\delta_{13}^{u}\right)_{L L}=\left(\delta_{31}^{u}\right)_{L L} \simeq 2.3 \times 10^{-3} \Delta a_{L}^{q}, \\
& \left(\delta_{23}^{u}\right)_{L L}=\left(\delta_{32}^{u}\right)_{L L} \simeq-4.4 \times 10^{-2} \Delta a_{L}^{q}, \\
& \left(\delta_{12}^{u}\right)_{R R}=\left(\delta_{21}^{u}\right)_{R R} \simeq-4.5 \times 10^{-4} \Delta a_{R}^{u}, \\
& \left(\delta_{13}^{u}\right)_{R R}=\left(\delta_{31}^{u}\right)_{R R} \simeq 4.8 \times 10^{-3} \Delta a_{R}^{u}, \\
& \left(\delta_{23}^{u}\right)_{R R}=\left(\delta_{32}^{u}\right)_{R R} \simeq 9.3 \times 10^{-2} \Delta a_{R}^{u} .
\end{aligned}
$$




\section{Up quark sector $(L R)$ :}

$$
\begin{aligned}
& \left(\delta_{11}^{u}\right)_{L R} \simeq 10^{-6}\left[3.8\left(\tilde{A}_{a}^{u}-\tilde{A}_{b}^{u}-\tilde{A}_{b^{\prime}}^{u}+2 \tilde{A}_{c}^{u}\right)\right]\left(\frac{500 \mathrm{GeV}}{m_{\tilde{q}}}\right), \\
& \left(\delta_{22}^{u}\right)_{L R} \simeq 10^{-3}\left[-1.4\left(\tilde{A}_{a}^{u}-\tilde{A}_{b}^{u}-\tilde{A}_{b^{\prime}}^{u}\right)+7.7 \times 10^{-3} \tilde{A}_{c}^{u}\right]\left(\frac{500 \mathrm{GeV}}{m_{\tilde{q}}}\right), \\
& \left(\delta_{33}^{u}\right)_{L R} \simeq\left[0.35 \tilde{A}_{a}^{u}+6.8 \times 10^{-4} \tilde{A}_{b}^{u}+3.0 \times 10^{-3} \tilde{A}_{b^{\prime}}^{u}+1.7 \times 10^{-10} \tilde{A}_{c}^{u}\right]\left(\frac{500 \mathrm{GeV}}{m_{\tilde{q}}}\right), \\
& \left(\delta_{12}^{u}\right)_{L R} \simeq-\left(\delta_{21}^{u}\right)_{L R} \simeq 7.4 \times 10^{-5}\left(\tilde{A}_{a}^{u}-\tilde{A}_{b}^{u}-\tilde{A}_{b^{\prime}}^{u}+\tilde{A}_{c}^{u}\right)\left(\frac{500 \mathrm{GeV}}{m_{\tilde{q}}}\right), \\
& \left(\delta_{13}^{u}\right)_{L R} \simeq 10^{-4}\left[-7.9\left(\tilde{A}_{a}^{u}-\tilde{A}_{b}^{u}\right)-7.0 \times 10^{-2}\left(\tilde{A}_{b^{\prime}}^{u}-\tilde{A}_{c}^{u}\right)\right]\left(\frac{500 \mathrm{GeV}}{m_{\tilde{q}}}\right), \\
& \left(\delta_{31}^{u}\right)_{L R} \simeq 10^{-3}\left[-1.7\left(\tilde{A}_{a}^{u}-\tilde{A}_{b^{\prime}}^{u}\right)-3.3 \times 10^{-3}\left(\tilde{A}_{b}^{u}-\tilde{A}_{c}^{u}\right)\right]\left(\frac{500 \mathrm{GeV}}{m_{\tilde{q}}}\right), \\
& \left(\delta_{23}^{u}\right)_{L R} \simeq 10^{-2}\left[1.5\left(\tilde{A}_{a}^{u}-\tilde{A}_{b}^{u}\right)+1.3 \times 10^{-2} \tilde{A}_{b^{\prime}}^{u}+3.6 \times 10^{-5} \tilde{A}_{c}^{u}\right]\left(\frac{500 \mathrm{GeV}}{m_{\tilde{q}}}\right), \\
& \left(\delta_{32}^{u}\right)_{L R} \simeq 10^{-2}\left[-3.2\left(\tilde{A}_{a}^{u}-\tilde{A}_{b^{\prime}}^{u}\right)-6.4 \times 10^{-3} \tilde{A}_{b}^{u}-1.7 \times 10^{-5} \tilde{A}_{c}^{u}\right] \quad\left(\frac{500 \mathrm{GeV}}{m_{\tilde{q}}}\right) .
\end{aligned}
$$

Down quark sector ( $L L$ and $R R)$ :

$$
\begin{aligned}
& \left(\delta_{12}^{d}\right)_{L L}=\left(\delta_{21}^{d}\right)_{L L} \simeq 1.2 \times 10^{-4} \Delta a_{L}^{q}, \\
& \left(\delta_{13}^{d}\right)_{L L}=\left(\delta_{31}^{d}\right)_{L L} \simeq 7.8 \times 10^{-3} \Delta a_{L}^{q}, \\
& \left(\delta_{23}^{d}\right)_{L L}=\left(\delta_{32}^{d}\right)_{L L} \simeq-1.5 \times 10^{-2} \Delta a_{L}^{q}, \\
& \left(\delta_{12}^{d}\right)_{R R}=\left(\delta_{21}^{d}\right)_{R R} \simeq-1.7 \times 10^{-1} \Delta a_{R}^{d}, \\
& \left(\delta_{13}^{d}\right)_{R R}=\left(\delta_{31}^{d}\right)_{R R} \simeq 1.4 \times 10^{-1} \Delta a_{R}^{d}, \\
& \left(\delta_{23}^{d}\right)_{R R}=\left(\delta_{32}^{d}\right)_{R R} \simeq 4.7 \times 10^{-1} \Delta a_{R}^{d} .
\end{aligned}
$$


Down quark sector $(L R)$ :

$$
\begin{aligned}
& \left(\delta_{11}^{d}\right)_{L R} \simeq 10^{-6}\left[6.6\left(\tilde{A}_{a}^{d}-\tilde{A}_{b}^{d}-\tilde{A}_{b^{\prime}}^{d}+2 \tilde{A}_{c}^{d}\right)\right]\left(\frac{500 \mathrm{GeV}}{m_{\tilde{q}}}\right), \\
& \left(\delta_{22}^{d}\right)_{L R} \simeq 10^{-5}\left[-4.1\left(\tilde{A}_{a}^{d}-\tilde{A}_{b^{\prime}}^{d}\right)+11 \tilde{A}_{b}^{d}+1.5 \tilde{A}_{c}^{d}\right]\left(\frac{500 \mathrm{GeV}}{m_{\tilde{q}}}\right), \\
& \left(\delta_{33}^{d}\right)_{L R} \simeq 10^{-3}\left[2.3 \tilde{A}_{a}^{d}+1.5 \times 10^{-3} \tilde{A}_{b}^{d}+3.5 \tilde{A}_{b^{\prime}}^{d}+1.4 \times 10^{-4} \tilde{A}_{c}^{d}\right]\left(\frac{500 \mathrm{GeV}}{m_{\tilde{q}}}\right), \\
& \left(\delta_{12}^{d}\right)_{L R} \simeq 10^{-5}\left[2.1\left(\tilde{A}_{a}^{d}-\tilde{A}_{b}^{d}-\tilde{A}_{b^{\prime}}^{d}+\tilde{A}_{c}^{d}\right)\right]\left(\frac{500 \mathrm{GeV}}{m_{\tilde{q}}}\right), \\
& \left(\delta_{21}^{d}\right)_{L R} \simeq 10^{-5}\left[-1.3\left(\tilde{A}_{a}^{d}-\tilde{A}_{b^{\prime}}^{d}\right)+3.4\left(\tilde{A}_{b}^{d}-\tilde{A}_{c}^{d}\right)\right]\left(\frac{500 \mathrm{GeV}}{m_{\tilde{q}}}\right), \\
& \left(\delta_{13}^{d}\right)_{L R} \simeq 10^{-5}\left[-1.8\left(\tilde{A}_{a}^{d}-\tilde{A}_{b}^{d}\right)-2.8\left(\tilde{A}_{b^{\prime}}^{d}-\tilde{A}_{c}^{d}\right)\right]\left(\frac{500 \mathrm{GeV}}{m_{\tilde{q}}}\right), \\
& \left(\delta_{31}^{d}\right)_{L R} \simeq 10^{-4}\left[-8.4\left(\tilde{A}_{a}^{d}-\tilde{A}_{b^{\prime}}^{d}\right)-10^{-3}\left(5.6 \tilde{A}_{b}^{d}-6.1 \tilde{A}_{c}^{d}\right)\right]\left(\frac{500 \mathrm{GeV}}{m_{\tilde{q}}}\right), \\
& \left(\delta_{23}^{d}\right)_{L R} \simeq 10^{-5}\left[3.5 \tilde{A}_{a}^{d}-9.3 \tilde{A}_{b}^{d}+5.3 \tilde{A}_{b^{\prime}}^{d}+0.52 \tilde{A}_{c}^{d}\right]\left(\frac{500 \mathrm{GeV}}{m_{\tilde{q}}}\right), \\
& \left(\delta_{32}^{d}\right)_{L R} \simeq 10^{-3}\left[-2.7\left(\tilde{A}_{a}^{d}-\tilde{A}_{b^{\prime}}^{d}\right)-1.8 \times 10^{-3} \tilde{A}_{b}^{d}-6.7 \times 10^{-5} \tilde{A}_{c}^{d}\right]\left(\frac{500 \mathrm{GeV}}{m_{\tilde{q}}}\right) .
\end{aligned}
$$

\begin{tabular}{|c||c|c|}
\hline & Exp. bound & $Q_{6}$ Model \\
\hline \hline$\sqrt{\left|\operatorname{Re}\left(\delta_{12}^{d}\right)_{L L, R R}^{2}\right|}$ & $4.0 \times 10^{-2} \tilde{m}_{\tilde{q}}$ & $(L L) 1.2 \times 10^{-4} \Delta a_{L}^{q},(R R) 1.7 \times 10^{-1} \Delta a_{R}^{d}$ \\
\hline$\sqrt{\left|\operatorname{Re}\left(\delta_{12}^{d}\right)_{L L}\left(\delta_{12}^{d}\right)_{R R}\right|}$ & $2.8 \times 10^{-3} \tilde{m}_{\tilde{q}}$ & $4.5 \times 10^{-3} \sqrt{\Delta a_{L}^{q} \Delta a_{R}^{d}}$ \\
\hline$\sqrt{\left|\operatorname{Re}\left(\delta_{12}^{d}\right)_{L R}^{2}\right|}$ & $4.4 \times 10^{-3} \tilde{m}_{\tilde{q}}$ & $\sim 2 \times 10^{-5}\left(\tilde{A}_{a}^{d}-\tilde{A}_{b}^{d}-\tilde{A}_{b^{\prime}}^{d}+\tilde{A}_{c}^{d}\right) \tilde{m}_{\tilde{q}}^{-1}$ \\
\hline$\sqrt{\left|\operatorname{Re}\left(\delta_{13}^{d}\right)_{L L, R R}^{2}\right|}$ & $9.8 \times 10^{-2} \tilde{m}_{\tilde{q}}$ & $(L L) 7.8 \times 10^{-3} \Delta a_{L}^{q},(R R) 1.4 \times 10^{-1} \Delta a_{R}^{d}$ \\
\hline$\sqrt{\left|\operatorname{Re}\left(\delta_{13}^{d}\right)_{L L}\left(\delta_{13}^{d}\right)_{R R}\right|}$ & $1.8 \times 10^{-2} \tilde{m}_{\tilde{q}}$ & $3.4 \times 10^{-2} \sqrt{\Delta a_{L}^{q} \Delta a_{R}^{d}}$ \\
\hline$\sqrt{\left|\operatorname{Re}\left(\delta_{13}^{d}\right)_{L R}^{2}\right|}$ & $3.3 \times 10^{-2} \tilde{m}_{\tilde{q}}$ & $\sim 2 \times 10^{-5}\left(\tilde{A}_{a}^{d}-\tilde{A}_{b}^{d}+\tilde{A}_{b^{\prime}}^{d}-\tilde{A}_{c}^{d}\right) \tilde{m}_{\tilde{q}}^{-1}$ \\
\hline$\sqrt{\left|\operatorname{Re}\left(\delta_{12}^{u}\right)_{L L, R R}^{2}\right|}$ & $1.0 \times 10^{-1} \tilde{m}_{\tilde{q}}$ & $(L L) 1.0 \times 10^{-4} \Delta a_{L}^{q},(R R) 4.5 \times 10^{-4} \Delta a_{R}^{u}$ \\
\hline$\sqrt{\left|\operatorname{Re}\left(\delta_{12}^{u}\right)_{L L}\left(\delta_{12}^{u}\right)_{R R}\right|}$ & $1.7 \times 10^{-2} \tilde{m}_{\tilde{q}}$ & $2.1 \times 10^{-4} \sqrt{\Delta a_{L}^{q} \Delta a_{R}^{u}}$ \\
\hline$\sqrt{\left|\operatorname{Re}\left(\delta_{12}^{u}\right)_{L R}^{2}\right|}$ & $3.1 \times 10^{-2} \tilde{m}_{\tilde{q}}$ & $\sim 7 \times 10^{-5}\left(\tilde{A}_{a}^{u}-\tilde{A}_{b}^{u}-\tilde{A}_{b^{\prime}}^{u}+\tilde{A}_{c}^{u}\right) \tilde{m}_{\tilde{q}}^{-1}$ \\
\hline$\left|\left(\delta_{23}^{d}\right)_{L L, R R}\right|$ & $8.2 \tilde{m}_{\tilde{q}}^{2}$ & $(L L) 1.5 \times 10^{-2} \Delta a_{L}^{q},(R R) 4.7 \times 10^{-1} \Delta a_{R}^{d}$ \\
\hline$\left|\left(\delta_{23}^{d}\right)_{L R}\right|$ & $1.6 \times 10^{-2} \tilde{m}_{\tilde{q}}^{2}$ & $\sim 5 \times 10^{-5}\left(\tilde{A}_{a}^{d}-\tilde{A}_{b}^{d}+\tilde{A}_{b^{\prime}}^{d}+0.1 \tilde{A}_{c}^{d}\right) \tilde{m}_{\tilde{q}}^{-1}$ \\
\hline
\end{tabular}

TABLE II: Experimental bounds on $\delta$ 's and their theoretical values in $Q_{6}$ model, where the parameter $\tilde{m}_{\tilde{q}}$ denotes $m_{\tilde{q}} / 500 \mathrm{GeV}$, and $\Delta a_{L, R}$ and $\tilde{A}$ are given in (62).

In Table II and III, theoretical values of certain $\delta$ 's calculated above and their experimental bounds are summarized. We see from the tables that to satisfy the experimental 


\begin{tabular}{|c||c|c|}
\hline & Exp. bound & $Q_{6}$ Model \\
\hline \hline$\left|\left(\delta_{12}^{e}\right)_{L L}\right|$ & $4.0 \times 10^{-5} \tilde{m}_{\tilde{\ell}}^{2}$ & $4.9 \times 10^{-3} \Delta a_{L}^{\ell}$ \\
\hline$\left|\left(\delta_{12}^{e}\right)_{R R}\right|$ & $9 \times 10^{-4} \tilde{m}_{\tilde{\ell}}^{2}$ & $8.4 \times 10^{-8} \Delta a_{R}^{e}$ \\
\hline$\left|\left(\delta_{12}^{e}\right)_{L R}\right|$ & $8.4 \times 10^{-7} \tilde{m}_{\tilde{\ell}}^{2}$ & $5.1 \times 10^{-6}\left(\tilde{A}_{b^{\prime}}^{e}-\tilde{A}_{c}^{e}\right) \tilde{m}_{\tilde{\ell}}^{-1}$ \\
\hline$\left|\left(\delta_{13}^{e}\right)_{L L}\right|$ & $2 \times 10^{-2} \tilde{m}_{\tilde{\ell}}^{2}$ & $1.7 \times 10^{-5} \Delta a_{L}^{\ell}$ \\
\hline$\left|\left(\delta_{13}^{e}\right)_{R R}\right|$ & $3 \times 10^{-1} \tilde{m}_{\tilde{\ell}}^{2}$ & $5.9 \times 10^{-2} \Delta a_{R}^{e}$ \\
\hline$\left|\left(\delta_{13}^{e}\right)_{L R}\right|$ & $1.7 \times 10^{-2} \tilde{m}_{\tilde{\ell}}^{2}$ & $3.1 \times 10^{-7}\left(\tilde{A}_{b}^{e}-\tilde{A}_{b^{\prime}}^{e}\right) \tilde{m}_{\tilde{\ell}}^{-1}$ \\
\hline$\left|\left(\delta_{23}^{e}\right)_{L L}\right|$ & $2 \times 10^{-2} \tilde{m}_{\tilde{\ell}}^{2}$ & $8.4 \times 10^{-8} \Delta a_{L}^{\ell}$ \\
\hline$\left|\left(\delta_{23}^{e}\right)_{R R}\right|$ & $3 \times 10^{-1} \tilde{m}_{\tilde{\ell}}^{2}$ & $1.4 \times 10^{-6} \Delta a_{R}^{e}$ \\
\hline$\left|\left(\delta_{23}^{e}\right)_{L R}\right|$ & $1 \times 10^{-2} \tilde{m}_{\tilde{\ell}}^{2}$ & $1.5 \times 10^{-9}\left(\tilde{A}_{b}^{e}-\tilde{A}_{b^{\prime}}^{e}\right) \tilde{m}_{\tilde{\ell}}^{-1}$ \\
\hline$\left|\left(\delta_{23}^{e}\right)_{L L}\left(\delta_{13}^{e}\right)_{L L}\right|$ & $1 \times 10^{-4} \tilde{m}_{\tilde{\ell}}^{2}$ & $1.4 \times 10^{-12}\left(\Delta a_{L}^{\ell}\right)^{2}$ \\
\hline$\left|\left(\delta_{23}^{e}\right)_{R R}\left(\delta_{13}^{e}\right)_{R R}\right|$ & $9 \times 10^{-4} \tilde{m}_{\tilde{\ell}}^{2}$ & $8.4 \times 10^{-8}\left(\Delta a_{R}^{e}\right)^{2}$ \\
\hline$\left|\left(\delta_{23}^{e}\right)_{L L}\left(\delta_{13}^{e}\right)_{R R}\right|$ & $2 \times 10^{-5} \tilde{m}_{\tilde{\ell}}^{2}$ & $5.0 \times 10^{-9} \Delta a_{L}^{\ell} \Delta a_{R}^{e}$ \\
\hline$\left|\left(\delta_{23}^{e}\right)_{R R}\left(\delta_{13}^{e}\right)_{L L}\right|$ & $2 \times 10^{-5} \tilde{m}_{\tilde{\ell}}^{2}$ & $2.4 \times 10^{-11} \Delta a_{L}^{\ell} \Delta a_{R}^{e}$ \\
\hline
\end{tabular}

TABLE III: Experimental bounds on $\delta$ 's and the theoretical values in $Q_{6}$ model, where the parameter $\tilde{m}_{\tilde{\ell}}$ denote $m_{\tilde{\ell}} / 100 \mathrm{GeV}$ and $\Delta a_{L, R}$ and $\tilde{A}$ are given in (62).

constraints, the SSB parameters $\Delta a_{R}^{d}, \Delta a_{L}^{\ell}, \tilde{A}_{b^{\prime}}^{e}$ and $\tilde{A}_{c}^{e}$ of the present $Q_{6}$ model should satisfy

$$
\Delta a_{R}^{d}<10^{-1}, \quad \Delta a_{L}^{\ell}<10^{-2}, \quad \tilde{A}_{b^{\prime}}^{e}-\tilde{A}_{c}^{e}<10^{-1}
$$

while the other SSB parameters are allowed to be of $O(1)$. So, one can fairly say that $Q_{6}$ symmetry can soften the SUSY flavor problem. Note also that the degree of degeneracy of the left-handed squark masses $\Delta a_{L}^{q}$ does not need to be very accurate. We find that because of the constraint on $\Delta a_{R}^{d}$ given in (69), $\Delta a_{L}^{q}<10^{+1}$ is sufficient to satisfy all the constraints. This has an important consequence for proton decay as we will see in the next section.

\section{PROTON DECAY}

In this section we consider proton decay, which is a process reflecting the flavor structure of a model.

\section{A. $Q_{6}$ invariant baryon and lepton number violating operators}

In supersymmetric models, the baryon and/or lepton number violating operators of lower dimensions are 27]: 
1. dimension-four $\mathrm{R}$ parity violating operators, and

2. dimension-five baryon and lepton number violating operators.

Both operators are controlled by the flavor structure of a model. As for the dimension-four $\mathrm{R}$ parity violating operators, $Q_{6}$ flavor symmetry in the model considered in the previous sections allows only lepton number violating operators:

$$
\begin{aligned}
& L L E^{c}: \lambda_{113} L_{I} L_{I} E_{3}^{c}+\lambda_{311} L_{3} L_{I} E_{I}^{c}+\lambda_{333} L_{3} L_{3} E_{3}^{c}+\lambda f_{I J K} L_{I} L_{J} E_{K}^{c}, \\
& L Q D^{c}: \lambda_{132}^{\prime} L_{I}\left(i \sigma^{2}\right)_{I J} Q_{3} D_{J}^{c}+\lambda_{123}^{\prime} L_{I}\left(\sigma^{1}\right)_{I J} Q_{J} D_{3}^{c} \text {, } \\
& H^{d} N H^{u}: \tilde{\lambda}_{312} H_{3}^{d} N_{I} H_{I}^{u}+\tilde{\lambda}_{333} H_{3}^{d} N_{3} H_{3}^{u}+\tilde{\lambda} f_{I J K} H_{I}^{d} N_{J} H_{K}^{u} \text {, } \\
& N N N: \tilde{\lambda}^{\prime} f_{I J K} N_{I} N_{J} N_{K}
\end{aligned}
$$

and those in which $L_{I}$ is replaced by $H_{I}^{d}$. Note that the baryon number violating operator $U^{c} D^{c} D^{c}$ is forbidden by $Q_{6}$ symmetry. Therefore, the dimension-four operators in the present model can not mediate proton decay.

We next look at dimension-five operators. The baryon number violating dimensionfive operators (which are allowed by $\mathrm{R}$ parity and $B-L$ symmetry) can be written as 27, 28, 52,53

$$
W_{5}=\frac{1}{M} \sum_{i, j, k, l=1 \sim 3}\left[\frac{1}{2} C_{L}^{i j k l} Q_{i} Q_{j} Q_{k} L_{l}+C_{R}^{i j k l} U_{i}^{c} E_{j}^{c} U_{k}^{c} D_{l}^{c}\right],
$$

where the first and the second term is called the LLLL and RRRR operator, respectively. In grand unified theories(GUTs), effective dimension-five operators can be generated by integrating out colored Higgs multiplets 27, 28], and therefore the size of the coefficients of the operators strongly depends the Yukawa matrices. For the minimal SUSY SU(5) GUT [53], one obtains

$$
M=M_{H_{C}}, C_{L}^{i j k l}=C_{R}^{i j k l}=Y_{U}^{i j} Y_{D}^{k l} \text { at } M_{G U T},
$$

where $M_{H_{C}}$ is the colored Higgs mass of order of the GUT scale, and $Y_{U}, Y_{D}$ are Yukawa coupling matrices appearing in the superpotential

$$
W_{Y}=\frac{1}{4} Y_{U}^{i j} \mathbf{1 0}^{i} \mathbf{1 0} 0^{j} H+\sqrt{2} Y_{D}^{i j} \mathbf{1 0}^{i} \overline{5}^{j} \bar{H} .
$$

Unfortunately, the minimal SUSY SU(5) GUT should be excluded by the decay mode $p \rightarrow K^{+} \bar{\nu}$ if the gauge coupling unification should be strictly satisfied [29].

If we do not assume any GUTs, the baryon number violating operators could be generated by some unknown Planck scale physics. In this case, the mass parameter $M$ in (711) is given by

$$
M=M_{P L}=2 \times 10^{18} \mathrm{GeV},
$$


while the coefficients $C_{L, R}^{i j k l}$ remain undetermined. So, the operators are supplied with a suppression factor $1 / M_{P L}$ which should be compared with $1 / M_{H_{C}} \simeq 1 / 10^{16} \mathrm{GeV}^{-1}$ in the GUT case. However, this suppression is not sufficient to keep the proton stable, unless that the coefficients $C^{\prime}$ 's are smaller than $\sim O\left(10^{-7}\right)$. An efficient tool to suppress or to forbid proton decay is symmetry. Many authors [23, 30, 54] have proposed a model in which both the gross structure of the baryon and lepton number violating operators and the structure of the Yukawa couplings are fixed by a single flavor symmetry and its breaking. If this is

realized, the flavor symmetry can be tested by proton decay, too. In the models of [23, 30, 54], a certain set of flavon fields is needed to form invariants, and the flavor symmetry is assumed to be broken at a superhigh energy scale. In contrast to these models, $Q_{6}$ flavor symmetry is broken (spontaneously and at most softly) only at a low energy scale which is comparable with the SUSY breaking scale. So, it is natural to assume that $Q_{6}$ flavor symmetry is intact at the Planck scale, too; we do not have to introduce flavon fields. Moreover, $Q_{6}$ is nonabelian, which can indeed reduce the number of independent coefficients drastically, as we will see now. We find that the relevant superpotential containing the baryon number violating $Q_{6}$ invariant dimension-five operators generated at the Planck scale can be written as

$W_{5}^{Q_{6}}=\frac{1}{M_{P L}} \sum_{I, J=1,2}\left[C_{L} Q_{I} Q_{I} Q_{3} L_{3}+C_{R}^{(1)} E_{I}^{c}\left(i \sigma^{2}\right)_{I J} U_{J}^{c} U_{3}^{c} D_{3}^{c}+C_{R}^{(2)} E_{I}^{c}\left(i \sigma^{2}\right)_{I J} U_{1}^{c} U_{2}^{c} D_{J}^{c}\right]$

where the superfields in (175) are in the flavor eigenstates. To obtain Eq.(75), we have not assumed any symmetries such as R-parity and $B-L$ except for $Q_{6}$ symmetry, where the $Q_{6}$ assignment is given in Table $\mathrm{I}$.

\section{B. Gross structure of the dimension-five operators and the lowest order approxi- mation}

As we can see from (75), $Q_{6}$ allows only three independent coefficients, $C_{L}, C_{R}^{(1)}$ and $C_{R}^{(2)}$. We will see moreover that the first term, the LLLL operator, gives the most dominant contribution to proton decay, while the RRRR operators can be neglected in the lowest order approximation. Consequently, the relative size of all the partial decay rates is fixed in this approximation, once the SSB sector is fixed. To begin with, we recall two basic facts:

1. Since the operators in the first two terms in $W_{5}^{Q_{6}}$ contain quark fields of the third generation in the flavor eigenstate, small mixing parameters appear when fields are rewritten in terms of the mass eigenstates, that is,

$$
\Phi_{3}^{f}=V_{3 I} \Phi_{I}^{m}, \quad I=1,2,
$$

where the subscripts $f$ and $m$ denote the flavor and mass eigenstates, respectively. The mixing parameters $V_{3 I}$ will be multiplied with the coefficients $C$ 's in the decay 
amplitudes, so that the condition on $C$ 's can be relaxed if $V_{3 I}$ are small (see ([36) (39) ). Note that within the framework of the MSSM, there is no such suppression.

2. In most of models, the universality of the SSB parameters at the GUT or Planck scale is assumed to suppress the SUSY contributions to FCNCs. This assumption has an important consequence that the gluino contributions to proton decay are negligibly small if all the squark masses are degenerate [55]. (In Fig. 3] we show a gaugino dressing diagram which contributes to an effective four-fermion operator.) However, in our case, we do not assume the universality. We have seen in the previous section that the degeneracy of the squark masses of the first two generations is almost exact due to $Q_{6}$ symmetry. Moreover, to suppress the SUSY contributions to FCNCs the degeneracy of the first two and third generations needs not to be accurate (see Eq. (69) and the discussions below). This means that the gluino dressing diagrams may not be negligible [56] in our case; we will have to investigate it.

We now argue that the first term, the LLLL operator, is the most dominant one. It is known that for the RRRR operators, the dominant contributions come from the gluino dressing diagrams. From the superpotential (75), we first observe that the second term in (75), the first RRRR operator, contains two quark fields of the third generation, implying that two small mixing parameters will be multiplied when going to the mass eigenstates. Further, the third term, the second RRRR term, contains only quark fields of the first two generation. That is, the gluino dressing contributions vanish because the squark masses of the first two generations are almost degenerate thanks to $Q_{6}$ symmetry. Thus, the LLLL operator is the only one which should be considered in the lowest order approximation, as we will do it in the following discussions.

Note that the LLLL operator contains two third generation fields $Q_{3}$ and $L_{3}$, but the $(3,1)$ element of the mixing matrix $U_{e L}$ (see (43) $)$ is equal to one, so that it does not act as a suppression factor. The dominant diagrams for the LLLL operator are those with gluino dressing. The zino and photino dressing diagrams have the same structure as the gluino ones, but they are negligibly small because the corresponding gauge couplings are small. So we ignore them in our calculations. We have calculated the higgsino dressing contributions and found that they can be neglected, $\operatorname{too}$, if $\tan \beta<10$. So we assume this to simplify our calculations. We may further approximate that the squark masses are diagonal in the super CKM basis. To see this, we recall that the squark mass squared in the super CKM basis can be written as

$$
\mathbf{m}_{a}^{2}=\left(\mathbf{m}_{f_{a}}\right)^{2}+\left(\begin{array}{cc}
\Delta_{L L}^{a} & \Delta_{L R}^{a *} \\
\Delta_{L R}^{a T} & \Delta_{R R}^{a T}
\end{array}\right), \quad a=u, d, e
$$

where $\mathbf{m}_{f_{a}}$ is the diagonal fermion mass matrix of the flavor $a$, and $\Delta$ 's are given in (60). As we can see from (64), (66) and (69), the nondiagonal elements are sufficiently small. 


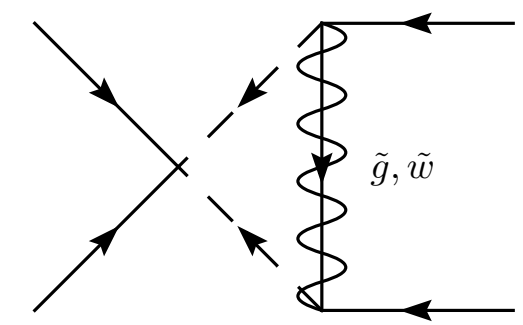

FIG. 3: One-loop diagrams contributing to the the effective four-fermi Lagrangian (78). We consider only gluino $(\tilde{g})$ and wino $(\tilde{w})$ dressings in the lowest order approximation.

The effective four-fermi Lagrangian $\mathcal{L}_{\text {eff }}$ can be obtained from the diagrams shown in Fig. 3, where as argued we consider only gluino and wino dressings. We find ${ }^{5}$

$$
\begin{aligned}
\mathcal{L}_{\mathrm{eff}}=\frac{1}{(4 \pi)^{2} M_{P L}} & {\left[\sum_{\substack{M=d, s \\
l=e, \mu}} C_{L L}(u d u e)^{1 M 1 l}\left(u d^{M}\right)\left(u e^{l}\right)+\sum_{\substack{M, N=d, s \\
l=e, \mu, \tau}} C_{L L}(u d d \nu)^{1 M N l}\left(u d^{M}\right)\left(d^{N} \nu^{l}\right)\right](78) } \\
C_{L L}(u d u e)^{1 M 1 l} & =\tilde{C}_{L L}^{\tilde{g}}(u d u e)^{1 M 1 l}+\tilde{C}_{L L}^{\tilde{w}}(u d u e)^{1 M 1 l} \\
C_{L L}(u d d \nu)^{1 M N l} & =\tilde{C}_{L L}^{\tilde{g}}(u d d \nu)^{1 M N l}+\tilde{C}_{L L}^{\tilde{w}}(u d d \nu)^{1 M N l}
\end{aligned}
$$

where $\tilde{C}^{\tilde{g}}$ and $\tilde{C}^{\tilde{w}}$ stand for the contributions coming from the gluino and wino dressing diagrams, respectively. These are explicitly calculated to be

$$
\begin{aligned}
\tilde{C}_{L L}^{\tilde{g}}(u d d \nu)^{1 M N l}= & 4 \pi \frac{4 \alpha_{3}}{3} \sum_{I=1,2} U_{u L}^{I 1} U_{d L}^{I M} U_{d L}^{3 N} U_{e L}^{3 l}\left(F^{\tilde{g}}\left(a_{L}^{q}, b_{L}^{q}\right)-F^{\tilde{g}}\left(a_{L}^{q}, a_{L}^{q}\right)\right), \\
\tilde{C}_{L L}^{\tilde{w}}(u d d \nu)^{1 M N l}= & 4 \pi \alpha_{2} \sum_{I=1,2}\left[U_{u L}^{I 1} U_{d L}^{I M} U_{d L}^{3 N} U_{e L}^{3 l}\left(F^{\tilde{w}}\left(a_{L}^{q}, a_{L}^{q}\right)+F^{\tilde{w}}\left(b_{L}^{q}, b_{L}^{\ell}\right)\right),\right. \\
& \left.-U_{u L}^{31} U_{d L}^{I M} U_{d L}^{I N} U_{e L}^{3 l}\left(F^{\tilde{w}}\left(a_{L}^{q}, b_{L}^{q}\right)+F^{\tilde{w}}\left(a_{L}^{q}, b_{L}^{\ell}\right)\right)\right], \\
\tilde{C}_{L L}^{\tilde{g}}(u d u e)^{1 M 1 l}= & -4 \pi \frac{4 \alpha_{3}}{3} \sum_{I=1,2} U_{u L}^{I 1} U_{d L}^{I M} U_{u L}^{31} U_{e L}^{3 l}\left(F^{\tilde{g}}\left(a_{L}^{q}, b_{L}^{q}\right)-F^{\tilde{g}}\left(a_{L}^{q}, a_{L}^{q}\right)\right), \\
\tilde{C}_{L L}^{\tilde{w}}(u d u e)^{1 M 1 l}= & -4 \pi \alpha_{2} \sum_{I=1,2}\left[U_{u L}^{I 1} U_{d L}^{I M} U_{u L}^{31} U_{e L}^{3 l}\left(F^{\tilde{w}}\left(a_{L}^{q}, a_{L}^{q}\right)+F^{\tilde{w}}\left(b_{L}^{q}, b_{L}^{\ell}\right)\right),\right. \\
& \left.-U_{u L}^{I 1} U_{d L}^{3 M} U_{u L}^{I 1} U_{e L}^{3 l}\left(F^{\tilde{w}}\left(a_{L}^{q}, b_{L}^{q}\right)+F^{\tilde{w}}\left(a_{L}^{q}, b_{L}^{\ell}\right)\right)\right],
\end{aligned}
$$

${ }^{5}$ We use the notation of $[57,58]$. 
where the loop functions are defined as [57, 58]

$$
\begin{aligned}
F^{\tilde{g}}\left(a_{L}^{q}, b_{L}^{q}\right) & =\frac{1}{m_{\tilde{g}}} \frac{1}{x_{g 1}-x_{g 3}}\left[\frac{x_{g 1} \ln x_{g 1}}{x_{g 1}-1}-\frac{x_{g 3} \ln x_{g 3}}{x_{g 3}-1}\right], \\
F^{\tilde{w}}\left(a_{L}^{q}, b_{L}^{\ell}\right) & =\frac{1}{m_{\tilde{w}}} \frac{1}{x_{w 1}-y_{w 3}}\left[\frac{x_{w 1} \ln x_{w 1}}{x_{w 1}-1}-\frac{y_{w 3} \ln y_{w 3}}{y_{w 3}-1}\right],
\end{aligned}
$$

with

$$
x_{g, w 1}=\frac{m_{\tilde{q}}^{2}}{m_{\tilde{g}, \tilde{w}}^{2}} a_{L}^{q}, x_{g, w 3}=\frac{m_{\tilde{q}}^{2}}{m_{\tilde{g}, \tilde{w}}^{2}} b_{L}^{q}, y_{w 3}=\frac{m_{\tilde{\ell}}^{2}}{m_{\tilde{w}}^{2}} b_{L}^{\ell} .
$$

We also use $\Delta_{Q} \equiv \Delta a_{L}^{q}=a_{L}^{q}-b_{L}^{q}$ (which is first introduced in (62) ) and

$$
r_{g}=\frac{m_{\tilde{q}}^{2}}{m_{\tilde{g}}^{2}}
$$

Then we calculate the decay amplitudes as a function of $x_{g 3}$ for given values of $r_{g} \Delta_{Q}$ and $y_{w 3}$, while for simplicity we assume the GUT relation between the wino and gluino mass ${ }^{6}$

$$
m_{\tilde{w}}=0.27 m_{\tilde{g}}
$$

The unitary matrices $U_{(u, d, e) L}$ in $C_{L L}$ are explicitly given in (36), (37) and (43), where the individual phases $\theta^{u, d}$ and $\theta_{3}^{u, d}$ in the quark sector are not fixed (see(26) ). However, it is found from (36) and (37) that the phase dependence of the combinations appearing in $C_{L L}$, that is, $U_{u L}^{I 1} U_{d L}^{I(1,2)}$ and $U_{(u, d) L}^{I 1} U_{(u, d) L}^{I 1}$, is small, and moreover the absolute size of the suppression factor $U_{(u, d) L}^{3 I}$ is independent of the phases. Therefore, in the following calculations we choose

$$
\theta^{u}=\theta_{3}^{u}=0, \theta_{3}^{d}-\theta^{d}=-1.25
$$

without significantly changing the results.

\section{Gluino versus wino contributions}

Before we calculate the decay amplitudes, we investigate the relative size of the gluino and wino contributions. As pointed out, to suppress the SUSY contributions to FCNCs in $Q_{6}$ model the degeneracy of the first two and third generations needs not to be exact. In fact, as we can see from Table III and Eq. (69), $\Delta_{Q} \leq 10$ is sufficient. ( $\Delta_{Q} \equiv \Delta a_{L}^{q}$ defined in (62) expresses the degree of the degeneracy of the squark masses.) If this is a case, the cancellation of the gluino contributions is no longer perfect [56], so that they may dominate over the wino ones. In Figs. 4,5] we present the results on the ratio $C^{\tilde{g}} / C^{\tilde{w}}$ as a function of $x_{g 3}$ for $r_{g} \Delta_{Q}=(10,1,0.1,0.01)$ and $y_{w 3}=10$ (right), 1 (left). Figs. 4 show the results

\footnotetext{
${ }^{6}$ If we change the GUT relation (84), the results below can change only slightly.
} 
on $C(u d d \nu)$ 's which control the size of the anti-neutrino modes, while Figs. [5 are those on $C(u d u e)$ 's for the decay amplitudes into a charged lepton. We can see furthermore that (except for $C(u d u e)^{121 l}$ shown in the last two figures of Figs. 5) the gluino contributions can become of the same order as the wino ones for $x_{g_{3}} \sim O(1)$ if $r_{g} \Delta_{Q} \gtrsim 1$. We can also see that the smaller the value of $r_{g} \Delta_{Q}$ is, the smaller are the gluino contributions, as it should be.

If the degeneracy of the squark masses is assumed, the gluino contributions vanish, and the wino contributions are then the dominant ones. This is assumed in most of the previous investigations on proton decay. Let us call the wino contribution under the assumption of the degeneracy (that is, $\left.\Delta_{Q}=0\right) C_{L L}(\cdots)_{M S S M}^{\tilde{w}}$, and compare it with $C_{L L}(\cdots)_{Q_{6}}^{\tilde{g}}$ for which $\Delta_{Q}$ may be different from zero. Since it will turn out that the decay mode $p \rightarrow K^{+} \bar{\nu}$ has the largest rate, we restrict ourselves to $C_{L L}(u d d \nu)^{112 l}$ (which gives the largest contribution to the decay mode). Fig . [6 shows the ratio $C_{L L}^{\tilde{g}}(u d d \nu)_{Q_{6}}^{112 l} / C_{L L}^{\tilde{w}}(u d d \nu)_{M S S M}^{112 l}$ as a function of $x_{g_{3}}$ for $r_{g} \Delta_{Q}=10$ and $y_{w_{3}}=1,10$. We see from the figure that the gluino contribution in $Q_{6}$ model is smaller than the wino ones of the MSSM, although the $S U(3)_{C}$ gauge coupling is larger than that the $S U(2)_{L}$ gauge coupling. This is due to the fact that the LLLL operator in (75) contains one $Q_{3}$ which requires a small mixing parameter to appear when expressed in terms of the mass eigenstates of the first two generations.

\section{Partial decay widths and their relative size}

Here we calculate the partial decay widths of the proton. The partial decay widths for the decay mode $p \rightarrow M \ell, \Gamma(p \rightarrow M \ell)$, can be written as [58]

$$
\Gamma(p \rightarrow M \ell)=\left(\frac{\mathcal{A} \beta_{p}}{(4 \pi)^{2} M_{P L}}\right)^{2} \frac{\left(m_{P}^{2}-m_{M}^{2}\right)^{2}}{32 \pi m_{P}^{3} f_{\pi}^{2}}|A m p(p \rightarrow M \ell)|^{2},
$$

with

$$
\begin{aligned}
A m p\left(p \rightarrow K^{+} \bar{\nu}\right) & =\kappa_{6} C_{L L}(u d d \nu)^{121 l}+\kappa_{2} C_{L L}(u d d \nu)^{112 l} \\
A m p\left(p \rightarrow \pi^{+} \bar{\nu}\right) & =\sqrt{2} \kappa_{5} C_{L L}(u d d \nu)^{111 l} \\
A m p\left(p \rightarrow K^{0} e_{l}^{+}\right) & =\kappa_{1} C_{L L}(u d u e)^{121 l} \\
A m p\left(p \rightarrow \pi^{0} e_{l}^{+}\right) & =\kappa_{5} C_{L L}(\text { udue })^{111 l}
\end{aligned}
$$

where $\kappa$ 's are defined by 58

$$
\begin{aligned}
\kappa_{1}=1+\frac{m_{P}}{m_{B}}(F-D)=0.70, & \kappa_{2}=1+\frac{m_{P}}{3 m_{B}}(3 F+D)=1.6, \\
\kappa_{3}=1+\frac{m_{P}}{m_{B}}(F+D)=2.0, & \kappa_{4}=2+\frac{2 m_{P}}{m_{B}} F=2.7, \\
\kappa_{5}=\frac{1}{\sqrt{2}}(1+F+D)=1.6, & \kappa_{6}=\frac{2 m_{P}}{3 m_{B}} D=0.4,
\end{aligned}
$$



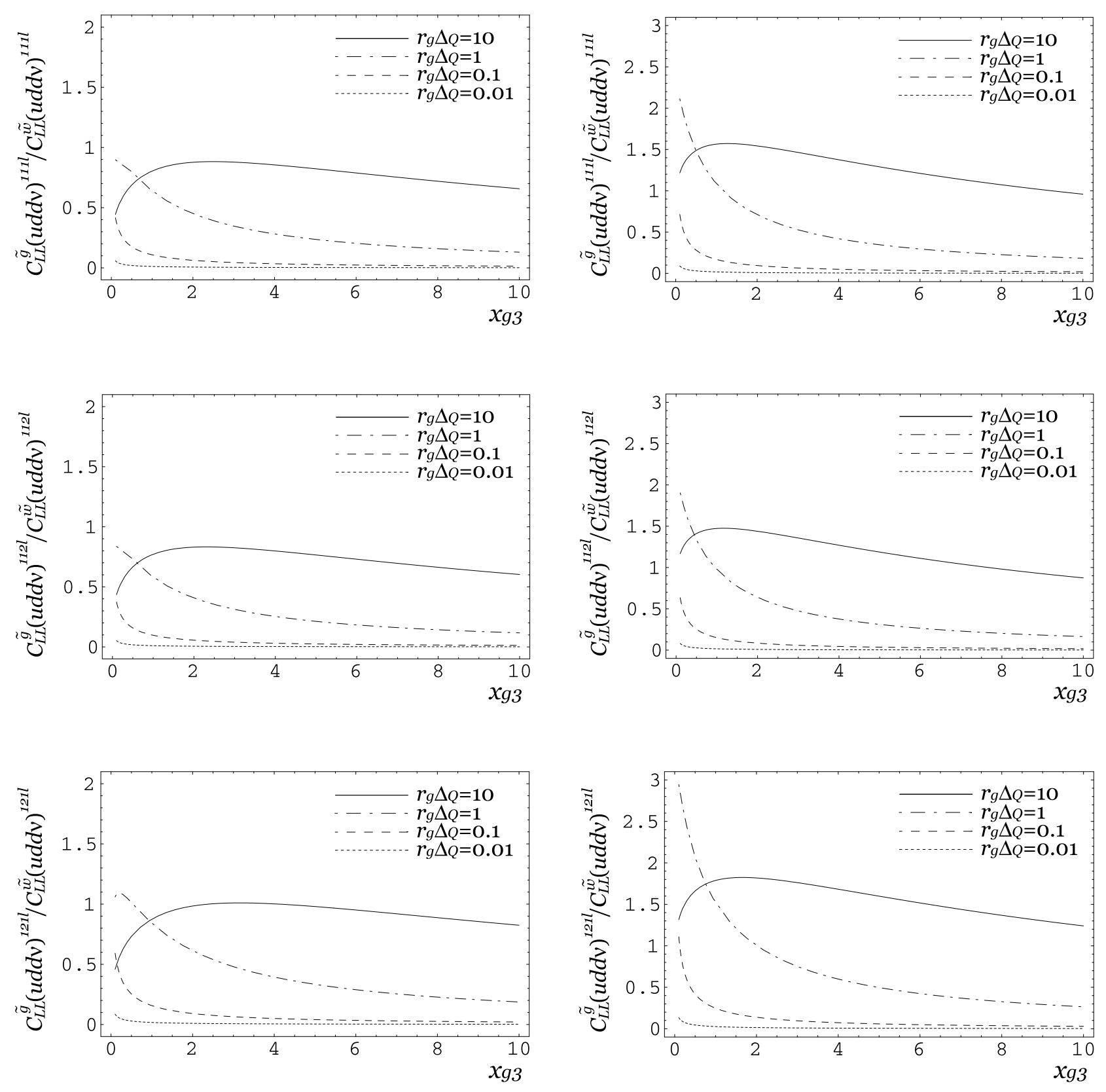

FIG. 4: The ratio $C^{\tilde{g}}(u d d \nu) / C^{\tilde{w}}(u d d \nu)$ is plotted as a function of $x_{g 3}$ for $r_{g} \Delta_{Q}=(10,1,0.1,0.01)$, which expresses the degree of the degeneracy of the squark masses, where $x_{g 3}$ is defined in (82). $C^{\tilde{g}}$ 's stand for the gluino contributions and $C^{\tilde{w}}$ 's for the wino ones, respectively. The figures in the left column correspond to $y_{w 3}=1$, and those in the right column to $y_{w 3}=10$. The corresponding amplitudes are responsible for the anti-neutrino modes.

and

$$
\begin{aligned}
D & =0.81, F=0.44, \beta_{p}=0.003 \mathrm{GeV}^{3}, f_{\pi}=131 \mathrm{MeV}, \\
m_{P} & =938 \mathrm{MeV}, m_{B}=1150 \mathrm{MeV}, m_{K}=495 \mathrm{MeV}, m_{\pi}=140 \mathrm{MeV} .
\end{aligned}
$$



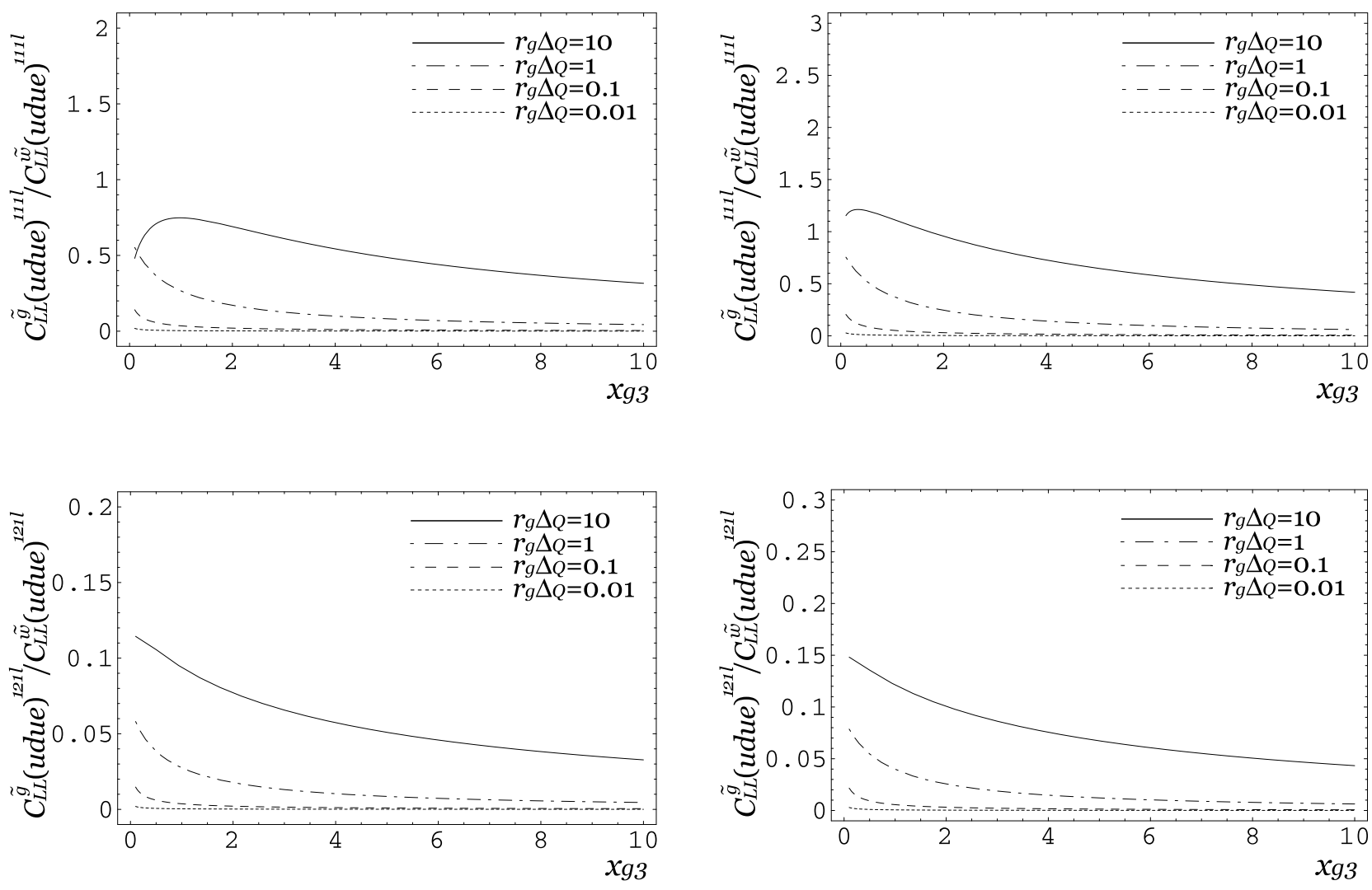

FIG. 5: The same as Fig. 4 for the charged lepton modes.

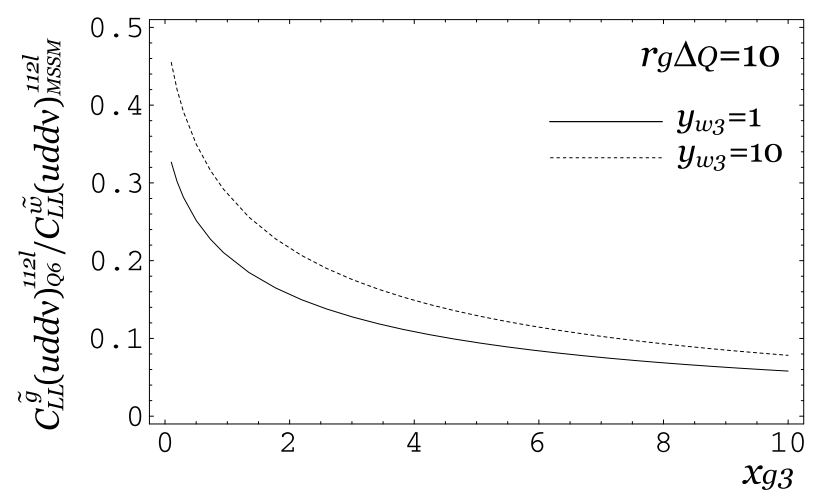

FIG. 6: Ratio of $C_{L L}^{\tilde{g}}(u d d \nu)_{Q_{6}}^{112 l}$ to $C_{L L}^{\tilde{w}}(u d d \nu)_{M S S M}^{112 l}$ as a function of $x_{g 3}$, where $r_{g} \Delta_{Q}=10$ is assumed for $C_{L L}^{\tilde{g}}(u d d \nu)_{Q_{6}}^{112 l}$, where $x_{g 3}$ is defined in (82). $C_{L L}(u d d \nu)^{112 l}$ is the largest coefficient for $p \rightarrow K^{+} \bar{\nu}$, and the degeneracy of the squark masses $\left(\Delta_{Q}=0\right)$ is assumed for $C_{L L}^{\tilde{w}}(u d d \nu)_{M S S M}^{112 l}$.

Here, $D, F$ stand for the coupling constants for the interaction between baryons and mesons, $\beta_{p}$ for the hadronic matrix element, $f_{\pi}$ for the pion decay constant, $m_{P}$ for the proton mass, $m_{B}$ for the averaged baryon mass, and $m_{K}$ and $m_{\pi}$ for the Kaon and pion mass, respectively. Further, $\mathcal{A}$ is the renormalization $\operatorname{group}(\mathrm{RG})$ enhancement factor for the coefficient $C_{L}$ 
[28, 53], which in our case becomes

$$
\mathcal{A}=10.5 \quad \text { (for the MSSM), } 12 \quad \text { (for } Q_{6} \text { model) }
$$

(Since $Q_{6}$ model contains four more Higgs doublets than in the case of the MSSM, the enhancement factor is slightly larger.)

As the first task, we would like to compare the decay rates in the MSSM and $Q_{6}$ model. By the MSSM decay rates we mean the decay rates which are obtained from the LLLL operators under the assumption of the degenerate squark masses (no gluino dressing). We also assume that all the coefficients of the operators are equal to the single constant $C_{L}^{M S S M}$, and that all the fields appearing in the operators are in the mass eigenstates (no mixing matrix). In Figs. [7] we plot for each decay mode, the experimental bound (thick solid line), the partial lifetime calculated in $Q_{6}$ model (solid line) and that in the MSSM (dotted line), where we assume

$$
C_{L}=C_{L}^{M S S M}=1, \quad m_{\tilde{q}}=1 \mathrm{TeV}
$$

The lifetime in $Q_{6}$ model is calculated from the sum of the gluino and wino contributions for $r_{g} \Delta_{Q}=0.01,0.1,1,10$, which corresponds to four solid lines. We first see from the figures that if $r_{g} \Delta_{Q}$ varies from 0.01 to 10 , a change of the life time about an order of magnitude can appear. We also see that the decay rates in $Q_{6}$ model is much more suppressed than those in the MSSM. Quantitatively, we find that the experimental bounds can be satisfied if the coefficient for the LLLL operator satisfies

$$
C_{L}<10^{-(4 \sim 5)}
$$

while $C_{L}^{M S S M}<10^{-(6 \sim 7)}$ should be satisfied for the case of the MSSM. That is, $Q_{6}$ flavor symmetry can suppress proton decay by four orders of magnitude (which can be also seen from the figures).

Next we would like to compare our results with those obtained in the minimal SUSY $S U(5)$ GUT. In our lowest order approximation, only $C_{L}$ is an independent coefficient, implying that the ratio of partial decay widths is independent of $C_{L}$. That is, in $Q_{6}$ model, the relative size of the partial decay rates is fixed (once the SSB sector is fixed). First we recall the case of the minimal SUSY SU(5) GUT [30, 53]. The superpotential for the baryon number violating effective dimension-five operators in this case is given by

$$
W_{5}^{S U(5)}=\frac{1}{2 M_{H_{C}}}\left[y_{u} y_{d l}\left(V^{*}\right)_{2 l}\left(Q_{1} Q_{1}\right)\left(Q_{2} L_{l}\right)+y_{c} y_{d l}\left(V^{*}\right)_{1 l}\left(Q_{2} Q_{2}\right)\left(Q_{1} L_{l}\right)\right]
$$

in the approximation that the third generation is dropped, where $y_{u, c}, y_{d l}$ are the diagonal Yukawa couplings of the corresponding quarks, $l=1,2$ is generation index (i.e., $d 1=d$ and $d 2=s$ ), and $V$ is the CKM matrix. In writing (96), a nontrivial assumption is made; the up quark Yukawa matrix is diagonal over the whole range of energies. Therefore, the 

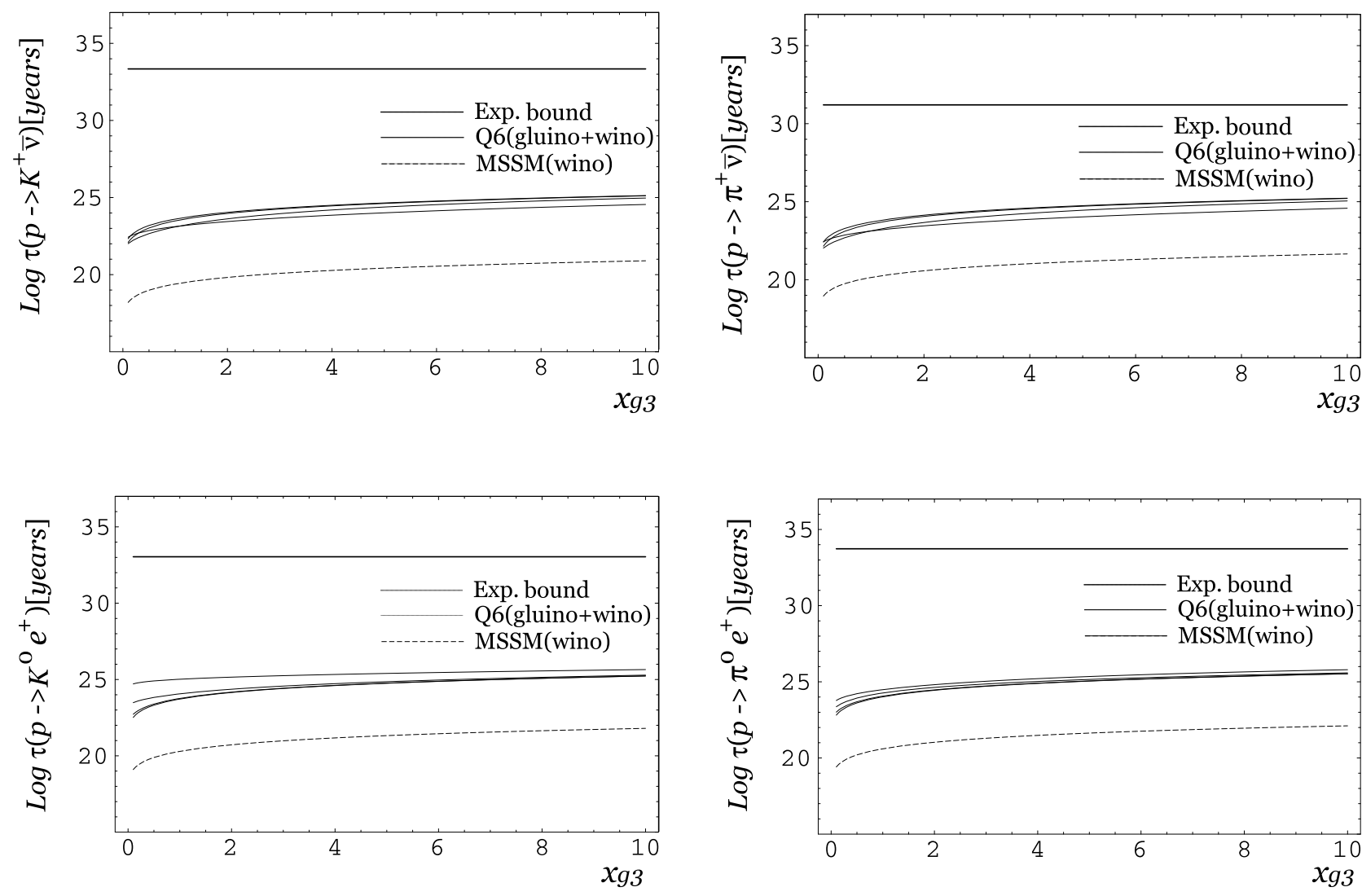

FIG. 7: Partial lifetime of the proton for each decay mode as a function of $x_{g 3}$ with $C_{L}=C_{L}^{M S S M}=$ $1, m_{\tilde{g}}=1 \mathrm{TeV}$. The experimental bound (thick solid line), the partial lifetime calculated in $Q_{6}$ model (solid line) and that in the MSSM (dotted line) are plotted. The lifetime in $Q_{6}$ model is calculated from the sum of the gluino and wino contributions for $r_{g} \Delta_{Q}=0.01,0.1,1,10$, which corresponds to four solid lines.

superpotential (96) is not a unique prediction of the the model. Under the assumption of the degeneracy of the squark masses, only the wino dressing diagrams contribute to the decay, and one finds 30,53$]$

$$
\begin{aligned}
\Gamma\left(p \rightarrow K^{+} \bar{\nu}\right) & =\gamma\left(m_{P}^{2}-m_{K}^{2}\right)^{2}\left|y_{c} y_{s} \sin ^{2} \theta_{C} \kappa_{3}\right|^{2}, \\
\Gamma\left(p \rightarrow \pi^{+} \bar{\nu}\right) & =\gamma\left(m_{P}^{2}-m_{\pi}^{2}\right)^{2}\left|2 y_{c} y_{s} \sin ^{2} \theta_{C} \tan \theta_{C} \sqrt{2} \kappa_{5}\right|^{2}, \\
\Gamma\left(p \rightarrow K^{0} e_{l}^{+}\right) & =\gamma\left(m_{P}^{2}-m_{K}^{2}\right)^{2}\left|y_{u} y_{d l}\left(V^{*}\right)_{2 l} \cos \theta_{C} \kappa_{1}\right|^{2}, \\
\Gamma\left(p \rightarrow \pi^{0} e_{l}^{+}\right) & =\gamma\left(m_{P}^{2}-m_{\pi}^{2}\right)^{2}\left|y_{u} y_{d l}\left(V^{*}\right)_{2 l} \sin \theta_{C} \kappa_{5}\right|^{2},
\end{aligned}
$$

where we have dropped the corresponding loop functions (82), and the common factor $\gamma$ is given by

$$
\gamma=\left(\frac{\mathcal{A} \beta_{p} \alpha_{2} \cos \theta_{C}}{\pi M_{H_{C}}}\right)^{2} \frac{1}{32 \pi m_{P}^{3} f_{\pi}^{2}} .
$$



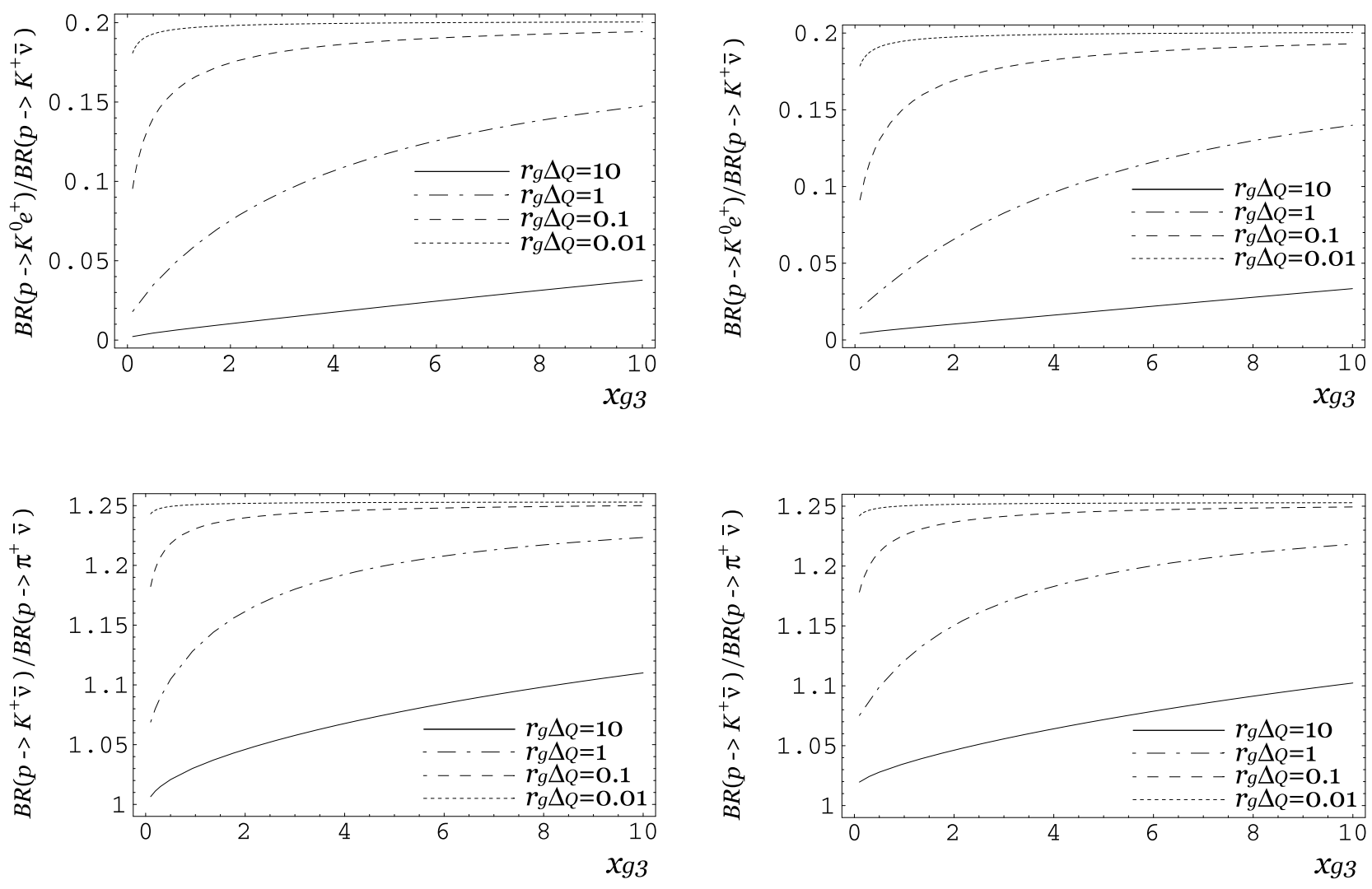

FIG. 8: Ratio of partial decay rates for each decay mode with $r_{g} \Delta_{Q}=(10,1,0.1,0.01)$. Figures in the left column are for $y_{w 3}=1$, and the right column for $y_{w 3}=10$.

From these partial decay widths, we obtain the relative decay widths in the minimal $S U(5)$ GUT [30, 53]:

$$
\begin{aligned}
& \frac{B\left(p \rightarrow K^{+} \bar{\nu}\right)}{B\left(p \rightarrow \pi^{+} \bar{\nu}\right)}=\frac{\left(m_{P}^{2}-m_{K}^{2}\right)^{2}}{\left(m_{P}^{2}-m_{\pi}^{2}\right)^{2}}\left(\frac{\kappa_{3}}{2 \sqrt{2} \kappa_{5} \tan \theta_{C}}\right)^{2} \simeq 2 \\
& \frac{B\left(p \rightarrow K^{0} e_{l}^{+}\right)}{B\left(p \rightarrow \pi^{0} e_{l}^{+}\right)}=\frac{\left(m_{P}^{2}-m_{K}^{2}\right)^{2}}{\left(m_{P}^{2}-m_{\pi}^{2}\right)^{2}}\left(\frac{\kappa_{1} \cos \theta_{C}}{\kappa_{5} \sin \theta_{C}}\right)^{2} \simeq 2 \\
& \frac{B\left(p \rightarrow K^{0} \mu^{+}\right)}{B\left(p \rightarrow K^{+} \bar{\nu}\right)}=\left(\frac{y_{u} \kappa_{1} \cos \theta_{C}}{y_{c} \kappa_{3} \sin ^{2} \theta_{C}}\right)^{2} \simeq 6 \times 10^{-4} \\
& \frac{B\left(p \rightarrow \pi^{0} \mu^{+}\right)}{B\left(p \rightarrow \pi^{+} \bar{\nu}\right)}=\left(\frac{y_{u} \cos ^{2} \theta_{C}}{2 \sqrt{2} y_{c} \sin ^{2} \theta_{C}}\right)^{2} \simeq 3 \times 10^{-4}
\end{aligned}
$$



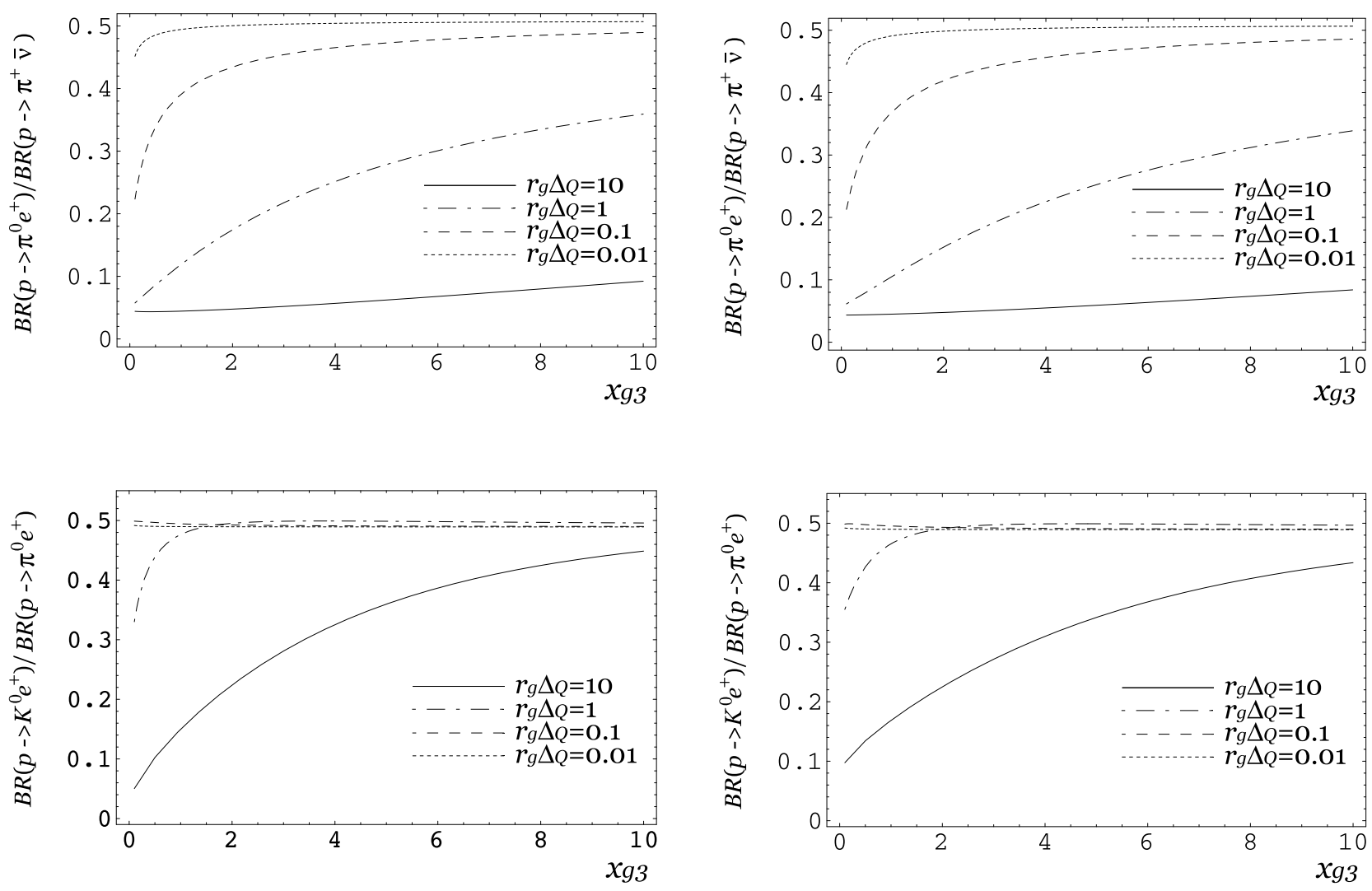

FIG. 9: Ratio of partial decay rates for each decay mode with $r_{g} \Delta_{Q}=(10,1,0.1,0.01)$. Figures in the left column are for $y_{w 3}=1$, and the right column for $y_{w 3}=10$.

The corresponding results for $Q_{6}$ model are found to be

$$
\begin{aligned}
& \frac{B\left(p \rightarrow K^{+} \bar{\nu}\right)}{B\left(p \rightarrow \pi^{+} \bar{\nu}\right)}=\frac{\left(m_{P}^{2}-m_{K}^{2}\right)^{2}}{\left(m_{P}^{2}-m_{\pi}^{2}\right)^{2}}\left(\frac{\kappa_{2} U_{d L}^{32}}{\sqrt{2} \kappa_{5} U_{d L}^{31}}\right)^{2} \simeq\left\{\begin{array}{l}
1.25 \\
1
\end{array}\right.
\end{aligned}
$$

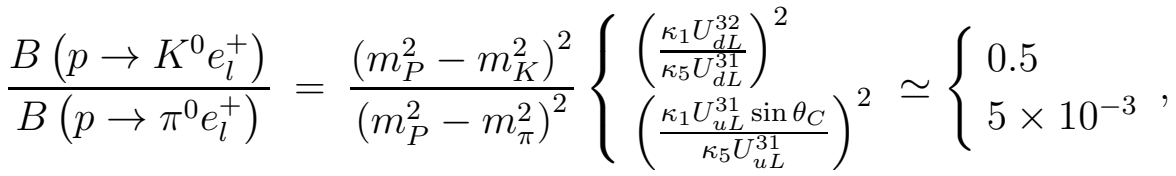

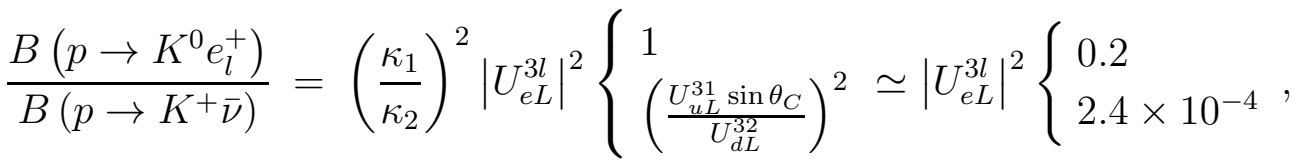

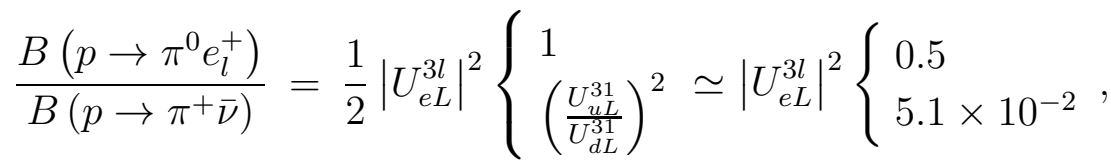

where as in the case of the minimal SUSY GUT we have suppressed the loop functions. The upper (lower) numbers on the right hand side correspond to the wino (gluino) contributions. Note that in contrast to the case of the minimal SUSY GUT the mixing parameters explicitly appear, reflecting the flavor structure of the present $Q_{6}$ model. The most remarkable difference is the charged lepton modes. As we see from (108) and (109), they are proportional 
to $\left|U_{e L}^{3 l}\right|^{2}$, where the unitary matrix $U_{e L}$ (which rotates the left-handed charged leptons) is explicitly given in (43). We find

$$
\begin{aligned}
\frac{B\left(p \rightarrow K^{0} \mu^{+}\right)}{B\left(p \rightarrow K^{0} e^{+}\right)} & =\frac{B\left(p \rightarrow \pi^{0} \mu^{+}\right)}{B\left(p \rightarrow \pi^{0} e^{+}\right)}=\frac{\left|U_{e L}^{32}\right|^{2}}{\left|U_{e L}^{31}\right|^{2}} \\
& =\left(\frac{m_{e}}{m_{\mu}}\right)^{2} \simeq 2.37 \times 10^{-5}
\end{aligned}
$$

The same ratio in the case of the minimal SUSY GUT becomes $B\left(p \rightarrow K^{0} \mu^{+}\right) \simeq 10^{3} \times$ $B\left(p \rightarrow K^{0} e^{+}\right)$if we use the superpotential (96). In Figs. 8 and 9 we plot four different

combinations of ratios as a function of $x_{g 3}$ for various values of $r_{g} \Delta_{Q}$ in $Q_{6}$ model. The figures on the left (right) side correspond to $y_{w_{3}}=1(10)$. From the figures, we find the hierarchical structure of the decay modes:

$$
B\left(p \rightarrow K^{0} e^{+}\right)<B\left(p \rightarrow \pi^{0} e^{+}\right)<B\left(p \rightarrow \pi^{+} \bar{\nu}\right)<B\left(p \rightarrow K^{+} \bar{\nu}\right)
$$

In the case of the minimal SUSY GUT we obtain instead

$$
B\left(p \rightarrow \pi^{0} e^{+}\right)<B\left(p \rightarrow K^{0} e^{+}\right)<<B\left(p \rightarrow \pi^{+} \bar{\nu}\right)<B\left(p \rightarrow K^{+} \bar{\nu}\right)
$$

Note, however, that although $B\left(p \rightarrow \pi^{0} e^{+}\right)<B\left(p \rightarrow K^{+} \bar{\nu}\right)$, they are basically in the same oder in the $Q_{6}$ model. That is, the $Q_{6}$ model predicts that once the decay mode $K^{+} \bar{\nu}$ is experimentally observed, then it is likely to observe the decay mode $\pi^{0} e^{+}$, too, in sharp contrast to the case of the minimal SUSY $S U(5)$ GUT.

\section{CONCLUSION}

Flavor symmetry can play important rolls in supersymmetric models [59]. We have investigated the SUSY contributions to FCNCs and to proton decay in a supersymmetric extension of the SM based on a binary dihedral family group $Q_{6}$. We have seen that the discrete low energy flavor symmetry $Q_{6}$ can be an alternative to the universality assumption of the soft supersymmetry breaking parameters. That is, the existence of a hidden sector, in which supersymmetry is assumed to be broken in a flavor blind manner, is not indispensable in this model. Therefore, a variety of supersymmetry breaking mechanisms may become phenomenologically viable.

It has turned out from our analysis on FCNCs that the degeneracy requirement of the soft scalar masses of the left-handed squarks in this model can be significantly relaxed. This has a considerable effect on the gluino-mediated one-loop amplitudes on proton decay. In most of the previous calculations, the degeneracy was assumed so that the gluino contributions are cancelled with each other, which implies that the wino contributions are the most dominant contributions. We have fond that the non-degenerate squarks can change the decay rate in the charged lepton modes by an order of magnidude. We have also found that in 
the lowest order approximation there is only one independent coefficient for dimension-five operators that lead to proton decay. Consequently, the relative partial decay rates in this approximation are fixed, reflecting the flavor structure dictated by $Q_{6}$.

Our main finding is that $Q_{6}$ flavor symmetry acts in such a way that the smallness of $\left(V_{\mathrm{MNS}}\right)_{e 3}$, the suppression of $\mu \rightarrow e+\gamma$, and the smallness of $B\left(p \rightarrow K^{0}\left(\pi^{0}\right)+\mu^{+}\right) / B\left(p \rightarrow K^{0}\left(\pi^{0}\right)+e^{+}\right)$have the same origin; the electron is much lighter than the muon and the tau. They in fact vanish in the $m_{e} \rightarrow 0$ limit.

\section{Acknowledgments}

We would like to thank J. Hisano, H. Nakano, T. Singai and K. Tobe for useful discussions. E.I is supported by Research Fellowship of the Japan Society for the Promotion of Science (JSPS) for Young Scientists (No.16-07971). This work is supported by the Grants-in-Aid for Scientific Research from the Japan Society for the Promotion of Science (\# 13135210).

[1] G. 't Hooft, "Naturalness, chiral symmetry, and spontaneous chiral symmetry breaking", in Recent developments in gauge theories, Cargèse, 1979.

[2] M. Veltman, Acta Phys. Pol. B12, 437 (1981).

[3] S.P. Martin, hep-ph/9709356

[4] D.J.H. Chung et al. Phys. Rept. 407, 1 (2005).

[5] S. Dimopoulos and D. Sutter, Nucl. Phys. B452, 496 (1995).

[6] L. Hall, V.A. Kostelecky and S. Raby, Nucl. Phys. B267, 415 (1986); F. Gabbiani and A. Masiero, Phys. Lett. B209, 289 (1988).

[7] J. Ellis and D.V. Nanopoulos, Phys. Lett. B110, 44 (1982); R. Barbieri and R. Gatto, Phys. Lett. B110, 211 (1982); B. Campbell, Phys. Rev. D 28, 209 (1983); M.J. Duncan, Nucl. Phys. B221, 285 (1983); J.F. Donoghue and H.P. Nilles and D. Wyler, Phys. Lett. B128, 55 (1983); J.-M. Gérard, W. Grimus, A. Raychaudhuri and G. Zoupanos, Phys. Lett. B140, 349 (1984).

[8] J. Ellis, S. Ferrara and D.V. Nanopoulos, Phys. Lett. B114, 231 (1982); W. Buchmüller and D. Wyler, Phys. Lett. B121, 321 (1983); J. Polchinski and M.B. Wise, Phys. Lett. B125, 393 (1983); F. del Aguila, M.B. Gavela, J.A. Grifols and A. Méndez, Phys. Lett. B126, 71 (1983); D.V. Nanopoulos and M. Srednicki, Phys. Lett. B128, 61 (1983).

[9] S. Bertolini, F. Borzumati, A. Masiero and G. Ridolfi, Nucl. Phys. B353, 591 (1991); R. Barbieri and G.F. Giudice, Phys. Lett. B309, 86 (1993).

[10] F. Gabbiani, E. Gabrielli, A. Masiero and L. Silvestrini, Nucl. Phys. B477, 321 (1996); S. Abel, S. Khalil and O. Lebedev, Nucl. Phys. B606, 151 (2001); M. Endo, M. Kakizaki and M. Yamaguchi, Phys. Lett. 583, 186 (2003); J. Hisano, Nucl. Phys. Proc. Suppl. 137, 169 (2004); J. Hisano and Y. Shimizu, Phys. Rev. D70, 093001 (2004). 
[11] S. Dimopoulos and H. Georgi, Nucl. Phys. B193, 150 (1981); N. Sakai, Z. Phys. C11, 153 (1981).

[12] M. Dine and A. E. Nelson, Phys. Rev. D48, 1277 (1993); M. Dine, A. E. Nelson and Y. Shirman, Phys. Rev. D51, 1362 (1995); For a review, G. F. Giudice and R. Rattazzi, Phys. Rept. 322, 419 (1999).

[13] L. Randall and R. Sundrum, Nucl. Phys. B557, 79 (1999); G. F. Giudice, M. A. Luty, H. Murayama and R. Rattazi, JHEP 12, 027 (1998).

[14] D. E. Kaplan, G. D. Kribs and M. Schmaltz, Phys. Rev. D62, 035010 (2000); Z. Chacko, M. A. Luty, A. E. Nelson and E. Ponton, JHEP 01, 003 (2000).

[15] M. Lanzagorta and G.G. Ross, Phys. Lett. B364, 163 (1995); P.M. Ferreira, I. Jack and D.R.T. Jones, Phys. Lett. B357, 359 (1995); S.A. Abel and B.C. Allanach, Phys. Lett. B415, 371 (1997); S.F. King and G.G. Ross, Nucl. Phys. B530, 3 (1998); I. Jack and D.R.T. Jones, Phys. Lett. B443, 177 (1998); G.K. Yeghiyan, M. Jurcisin and D.I. Kazakov, Mod. Phys. Lett. A14, 601 (1999); M. Jurcisin and D.I. Kazakov, Mod. Phys. Lett. A14, 671 (1999).

[16] A. Karch, T. Kobayashi, J. Kubo, and G. Zoupanos, Phys. Lett. B441, 235 (1998); M. Luty and R. Rattazzi, JHEP 11, 001 (1999).

[17] A. E. Nelson and M. J. Strassler, JHEP 09, (2000) 030; T. Kobayashi and H. Terao, Phys. Rev. D64, 075003 (2001).

[18] T. Kobayashi , H. Nakano and H. Terao, Phys. Rev. D65, 015006 (2002); T. Kobayashi, H. Nakano, T. Noguchi and H. Terao, Phys. Rev. D66 095011 (2002); JHEP 0302, 022 (2003).

[19] M. A. Luty and R. Sundrum, Phys. Rev. D65, 066004 (2002); Phys. Rev. D67, 045007 (2003).

[20] S.A. Abel and S.F. King, Phys. Rev. D59, 095010 (1999); T. Kobayashi and K. Yoshioka, Phys. Rev. D62, 115003 (2000); M. Bando, T. Kobayashi, T. Noguchi and K. Yoshioka, Phys. Rev. D63, 113017 (2001).

[21] J. Kubo and H. Terao, Phys. Rev. D66, 116003 (2002); K-S. Choi, K-Y. Choi and J.E. Kim, Phys. Rev. D68, 035003 (2003); K-Y. Choi, J.E. Kim and H. M. Lee, JHEP 0306, 040 (2003); Y. Kajiyama, J. Kubo and H. Terao, Phys. Rev. D69, 116006 (2004).

[22] L.J. Hall and H. Murayama, Phys. Rev. Lett. 75 (1995) 3985.

[23] C.D. Carone, L.J. Hall and H. Murayama, Phys. Rev. D53, 6282 (1996).

[24] K.S. Babu, T. Kobayashi and J. Kubo, Phys. Rev. D67, 075018 (2003); K. Hamaguchi, M. Kakizaki and M. Yamaguchi, Phys. Rev. D68, 056007 (2003); T. Kobayashi, J. Kubo and H. Terao, Phys. Lett. B568, 83 (2003); G. G. Ross, L. Velasco-Sevilla and Oscar Vives, Nucl. Phys. B692, 50 (2004); S. F. King and G. G. Ross, Phys. Lett. B520, 243 (2001); B574, 239 (2003); G. G. Ross and L. Velasco-Sevilla, Nucl.Phys. B653, 3 (2003); Ki-Y. Choi, Y. Kajiyama, J. Kubo and H.M. Lee, Phys. Rev. D70, 055004 (2004).

[25] N. Maekawa and T. Yamashita, JHEP 0407, 009 (2004); T. Yamashita, hep-ph/0503265.

[26] K.S. Babu and J. Kubo, Phys. Rev. D71, 056006 (2005). 
[27] N. Sakai and T. Yanagida, Nucl. Phys. B197, 533 (1982), S. Weinberg, Phys. Rev. D26, 287 (1982).

[28] J. Ellis, D.V. Nanopoulos and S. Rudaz, Nucl. Phys. B202, 43 (1982).

[29] H. Murayama and A. Pierce, Phys. Rev. D65, 055009 (2002).

[30] H. Murayama and D.B. Kaplan, Phys. Lett. B336, 221 (1994).

[31] P.H. Frampton and T.W. Kephart, Int. J. Mod. Phys. A10, 4689 (1995); P.H. Frampton and T.W. Kephart, Phys. Rev. D64, 086007 (2001).

[32] E. Ma and G. Rajasekaran, Phys. Rev. D64, 113012 (2001); E. Ma, Mod. Phys. Lett. A17, 627 (2002); 2361 (2002) .

[33] G. Seidl, hep-ph/0301044.

[34] J. Kubo, A. Mondragón, M. Mondragón and E. Rodríguez-Jáuregui, Prog. Theor. Phys. 109, 795 (2003); Erratum-ibid.114, 287 (2005); J. Kubo, Phys. Lett. B578, 156 (2004); Erratumibid. B619, 387 (2005).

[35] W. Grimus and L. Lavoura, Phys. Lett. B572, 76 (2003).

[36] T. Kobayashi, S. Raby and R-J. Zhang, Phys. Lett. B593, 262 (2004); Nucl. Phys. B704,3 (2005).

[37] S-L. Chen, M. Frigerio and E. Ma, Phys. Rev. D70, 073008 (2004); W. Grimus, A.S. Joshipura, S. Kaneko, L. Lavoura and M. Tanimoto, JHEP 0407, 078 (2004); G. Altarelli and F. Feruglio, New J. Phys. 6, 106 (2004); M. Frigerio, S. Kaneko, E. Ma and M. Tanimoto, Phys. Rev. D71, 011901 (2005); L. Lavoura and E. Ma, Mod. Phys. Lett. A20, 1217 (2005); S-L.Chen and E. Ma, Phys. Lett. B620, 151 (2005); C. Hagedorn and W. Rodejohann, JHEP 0507, 034 (2005); F. Caravaglios and S. Morisi, hep-ph/0503234 hep-ph/0503234 hep-ph/0510321; W. Grimus and L. Lavoura, JHEP 0508, 013 (2005); T. Teshima, hep-ph/0509094 N. Haba and K. Yoshioka, hep-ph/0511108

[38] K.S. Babu, I. Gogoladze and K. Wang, Phys. Lett. B560, 214 (2003); Nucl. Phys. B660, 322 (2003).

[39] J. Kubo, H. Okada and F. Sakamaki, Phys. Rev. D70, 036007 (2004).

[40] S. Weinberg, in Transactions of the New York Academy of Sciences, Series II, Vol. 38, 185 (1977); F. Wilczek and A. Zee, Phys. Rev. Lett. 42, 421 (1979).

[41] H. Fritzsch, Phys. Lett. B 73, 317 (1978); Nucl. Phys. B155, 189 (1979).

[42] Particle Data Group, Phys. Lett . B592, 1 (2004).

[43] H.D. Kim, S. Raby and L. Schradin, hep-ph/0401169.

[44] Heavy Flavor Averaging Group (HFAG), hep-ex/0412073

[45] K. Abe et al.(the Belle Collaboration), hep-ex/0506079.

[46] K. Harayama, N. Okamura, A.I. Sanda and Z-Z. Xing, Prog. Theor. Phys. 97, 781 (1997).

[47] P. Minkowski, Phys. Lett. B67, 421 (1977). T. Yanagida, in Proc, of the Workshop on the unified Theory and Baryon Number in the universe, ed. O. Sawada and A. Sugamoto, (KEK report 79-18, 1979); M. Gell-Mann, P. Ramond and R. Slansky, in Supergravity, ed P. van 
Nieuwenhuizen and d.Z. Freedman, (North Holland, Amsterdam, 1979); R. N. Mohapatra and G. Senjanovic, Phys. Rev. Lett. 44, 912 (1980).

[48] H. Minakata, H. Sugiyama, O. Yasuda, K. Inoue and F. Suekane, Phys. Rev. D68, 033017 (2003); Erratum-ibid. D70, 059901 (2004); O. Yasuda, hep-ph/0309333 H. Minakata, Nucl. Phys. B (Proc. Suppl.) 137, 74 (2004); P. Huber, M. Lindner, M. Rolinec, T. Schwetz, and W. Winter, Phys. Rev. D70, 073014 (2004); H. Sugiyama, O. Yasuda, F. Suekane and G.A. Horton-Smith, hep-ph/0409109.

[49] M. Maltoni, T. Schwetz, M.A. Tòrtalo and J.W.F. Valle, New J. Phys. 6, 122 (2004).

[50] H.V. Klapdor-Kleingrothaus et al., Eur. Phys. J. A12, 147 (2001); C.E. Aalseth et al., Phys. Atm. Nucl. 63, 1268 (2000); H.V. Klapdor-Kleingrothaus, A. Dietz, H.L. Harney and I.V. Krivosheina, Mod. Phys. Lett. A 16, 2409 (2001).

[51] D.N. Spergel et al., Astrophys. J. Suppl. 148, 175 (2003).

[52] S. Dimopoulos, S. Raby and F. Wilczek, Phys. Lett. B112, 133 (1982); P. Nath, A.H. Chamseddine and R. Arnowitt, Phys. Rev. D32, 2348 (1985).

[53] J. Hisano, H. Murayama and T. Yanagida, Nucl. Phys. B402, 46 (1993).

[54] V. Ben-Hamo and Y. Nir, Phys. Lett. B339, 77 (1994); M. Kakizaki and M. Yamaguchi, JHEP 0206, 032 (2002); R. Harnik, D.T. Larson, H. Murayama and M. Thormeier, Nucl. Phys. B706, 372 (2005).

[55] V.M. Belyaev and M.I. Vysotsky, Phys. Lett. B127, 215 (1983); J. Ellis, S. Hagelin, D.V. Nanopoulos and K. Tamvakis, Phys. Lett. B124, 484 (1983).

[56] S. Chadha, G.D. Coughlan, M. Daniel and G.G. Ross, Phys. Lett. B149, 477 (1984); J. Milutinović, P.B. Pal and G. Senjanović, Phys. Lett. B140, 324 (1984); M. McDonald and C.E. Vayonakis, Phys. Lett. B163, 148 (1985).

[57] T. Goto and T. Nihei and J. Arafune, Phys. Rev. D52, 505 (1995).

[58] T. Goto and T. Nihei, Phys. Rev. D59, 115009 (1999).

[59] E. Bilgin, B. Patt, D. Tucker-Smith and F. Wilczek, hep-ph/0509075, B. Patt, D. TuckerSmith and F. Wilczek, hep-ph/0509295. 